JOURNAL OF THE

AMERICAN MATHEMATICAL SOCIETY

Volume 16, Number 3, Pages 495-535

S 0894-0347(03)00424-7

Article electronically published on March 4, 2003

\title{
THE WEIL-PETERSSON METRIC AND VOLUMES OF 3-DIMENSIONAL HYPERBOLIC CONVEX CORES
}

\author{
JEFFREY F. BROCK
}

\section{INTRODUCTION}

Recent insights into the combinatorial geometry of Teichmüller space have shed new light on fundamental questions in hyperbolic geometry in 2 and 3 dimensions. Paradoxically, a coarse perspective on Teichmüller space appears to refine the analogy of Teichmüller geometry with the internal geometry of hyperbolic 3-manifolds first introduced and pursued by W. Thurston.

In this paper we develop such a coarse perspective on the Weil-Petersson metric on Teichmüller space by relating it to a graph of pair-of-pants decompositions of surfaces introduced by Hatcher and Thurston. This viewpoint generates a new connection between the Weil-Petersson geometry of Teichmüller space and the geometry of the convex core of a hyperbolic 3-manifold.

For simplicity, let $S$ be a closed oriented surface of negative Euler characteristic. A pants decomposition of $S$ is a maximal collection of distinct isotopy classes of pairwise disjoint, essential, simple closed curves on $S$. We say two distinct pants decompositions $P$ and $P^{\prime}$ are related by an elementary move if $P^{\prime}$ can be obtained from $P$ by replacing a curve $\alpha \in P$ by a curve $\beta$ intersecting $\alpha$ minimally (see Figure 3).

One obtains the pants graph $\mathbf{P}(S)$ by making each pants decomposition a vertex and joining two pants decompositions differing by an elementary move by an edge. Setting the length of each edge to $1, \mathbf{P}(S)$ becomes a metric space. We find the graph $\mathbf{P}(S)$ provides a combinatorial model for the coarse geometry of the WeilPetersson metric:

Theorem 1.1. The graph $\mathbf{P}(S)$ is naturally quasi-isometric to Teichmüller space with the Weil-Petersson metric.

The connection to hyperbolic 3-manifolds is simple to describe. By a theorem of Bers, a pair of points $(X, Y) \in \operatorname{Teich}(S) \times \operatorname{Teich}(S)$ naturally determines a quasiFuchsian hyperbolic 3-manifold $Q(X, Y) \cong S \times \mathbb{R}$ with $X$ and $Y$ in its conformal boundary at infinity. Its convex core, denoted core $(Q(X, Y))$, is the smallest convex subset of $Q(X, Y)$ carrying its fundamental group. The convex core is itself homeomorphic to $S \times I$ and carries all the essential geometric information about the manifold $Q(X, Y)$.

Received by the editors October 30, 2001.

2000 Mathematics Subject Classification. Primary 30F40; Secondary 30F60, 37F30.

Key words and phrases. Hyperbolic manifold, Kleinian group, pants decomposition, Teichmüller space, Weil-Petersson metric, limit set.

Research partially supported by NSF grant DMS-0072133 and an NSF postdoctoral fellowship.

(C)2003 American Mathematical Society 


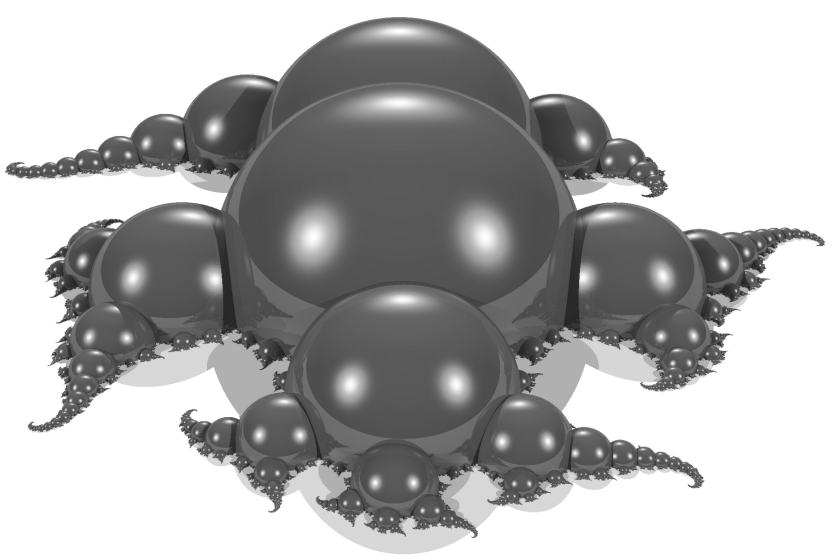

Figure 1 . The lift to $\mathbb{H}^{3}$ of a quasi-Fuchsian convex core boundary component.

Because $Q(X, Y)$ is obtained from the pair $(X, Y)$ by an analytic process (Bers's simultaneous uniformization), it is a central challenge in the study of hyperbolic 3manifolds to understand the geometry of $Q(X, Y)$ purely in terms of the geometry of $X$ and $Y$. Our main theorem proves a conjecture of Thurston that the following fundamental connection exists between convex core volume and the Weil-Petersson distance.

Theorem 1.2. The volume of the convex core of $Q(X, Y)$ is comparable to the Weil-Petersson distance $d_{\mathrm{WP}}(X, Y)$.

Here, comparability means that two quantities are equal up to a uniform additive and multiplicative error: i.e. there are constants $K_{1}>1$ and $K_{2}>0$ depending only on $S$ so that for any $(X, Y) \in \operatorname{Teich}(S) \times \operatorname{Teich}(S)$ we have

$$
\frac{d_{\mathrm{WP}}(X, Y)}{K_{1}}-K_{2} \leq \operatorname{vol}(\operatorname{core}(Q(X, Y))) \leq K_{1} d_{\mathrm{WP}}(X, Y)+K_{2} .
$$

Throughout the paper we will use the contraction $\operatorname{vol}(X, Y)=\operatorname{vol}(\operatorname{core}(Q(X, Y)))$ and the notation $\asymp$ to denote the comparability of two quantities; then Theorem 1.2 becomes

$$
d_{\mathrm{WP}}(X, Y) \asymp \operatorname{vol}(X, Y) \text {. }
$$

The volume of the convex core of a complete hyperbolic 3-manifold $M=\mathbb{H}^{3} / \Gamma$ is directly related to the lowest eigenvalue of the Laplacian on $M$ as well as the Hausdorff dimension of the limit set $\Lambda(\Gamma) \subset \widehat{\mathbb{C}}$, namely the complement of the invariant domain of discontinuity $\Omega(\Gamma) \subset \widehat{\mathbb{C}}$ where the action of Kleinian covering group $\Gamma \subset \mathrm{PSL}_{2}(\mathbb{C})$ for $M$ is properly discontinuous (see Figure 2 for two examples of limit set:11).

As an immediate application, Theorem 1.2 implies the following new relationship between these analytic invariants and the Weil-Petersson distance. Let $\lambda_{0}(X, Y)$ denote the lowest eigenvalue of the Laplacian on the quasi-Fuchsian hyperbolic 3manifold $Q(X, Y)=\mathbb{H}^{3} / \Gamma(X, Y)$ and let $D(X, Y)$ denote the Hausdorff dimension of the limit set of $\Gamma(X, Y)$.

\footnotetext{
${ }^{1}$ We have employed computer programs of Curt McMullen in our generation of Figures 1 and 2.
} 

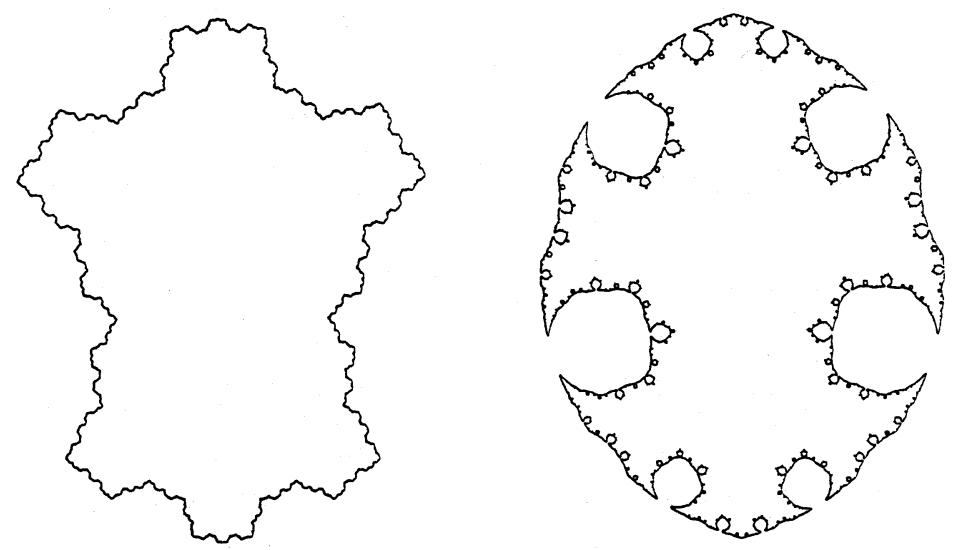

Figure 2. Limit sets for two quasi-Fuchsian groups.

Theorem 1.3. Given $S$ there are constants $K>0, C_{1}, C_{2}, C_{3}$, and $C_{4}>1$ so that if $d_{\mathrm{WP}}(X, Y)>K$, then

$$
\frac{C_{1}}{d_{\mathrm{WP}}(X, Y)^{2}} \leq \lambda_{0}(X, Y) \leq \frac{C_{2}}{d_{\mathrm{WP}}(X, Y)},
$$

and

$$
2-\frac{C_{3}}{d_{\mathrm{WP}}(X, Y)} \leq D(X, Y) \leq 2-\frac{C_{4}}{d_{\mathrm{WP}}(X, Y)^{2}}
$$

Proof. The relation $\lambda_{0}(X, Y)=D(X, Y)(2-D(X, Y))$ follows from a general result by D. Sullivan (see [Sul2, Thm. 2.17]), after applying Bowen's Theorem Bow that $D(X, Y) \geq 1$ with equality if and only if $X=Y$.

Theorem 1.2 may be rephrased to claim the existence of $K, K^{\prime}$ so that for $d_{\mathrm{WP}}(X, Y)>K$ we have

$$
\frac{d_{\mathrm{WP}}(X, Y)}{K^{\prime}} \leq \operatorname{vol}(X, Y) \leq K^{\prime} d_{\mathrm{WP}}(X, Y) .
$$

The theorem then follows from the double inequality

$$
\frac{c_{1}}{\operatorname{vol}(X, Y)^{2}} \leq \lambda_{0}(X, Y) \leq \frac{c_{2}}{\operatorname{vol}(X, Y)}
$$

(see [BC Main Thm.] and [Can1, Thm. A]) after collecting constants.

The pants graph. Since Theorem 1.2 relies directly on Theorem 1.1 we detail our coarse perspective on the Weil-Petersson metric.

To describe the nature of the quasi-isometry between the graph $\mathbf{P}(S)$ and the Weil-Petersson metric, we recall that by a theorem of Bers there is a constant $L>0$ depending only on $S$ so that for each $X \in \operatorname{Teich}(S)$ there is a pants decomposition $P$ so that

$$
\ell_{X}(\alpha)<L \text { for each } \alpha \in P,
$$

where $\ell_{X}(\alpha)$ denotes the length of the geodesic representative of $\alpha$ in the hyperbolic metric on $X$. 


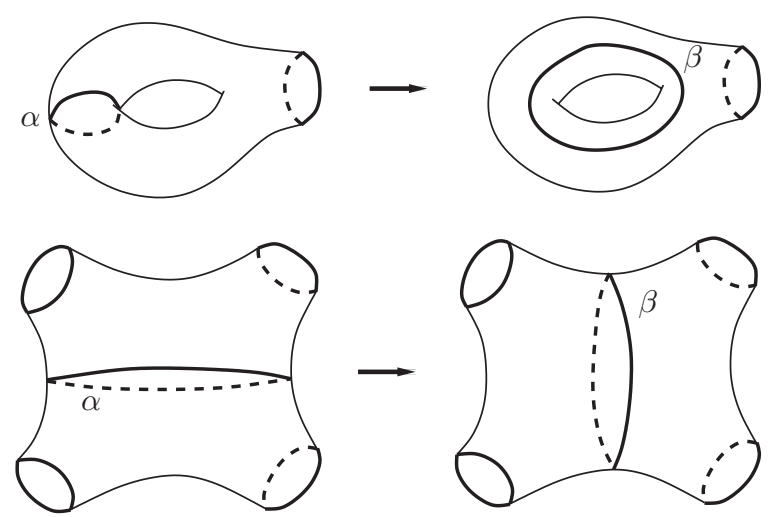

FiguRE 3. Elementary moves on pants decompositions.

If $P$ is a pants decomposition, we denote by $V_{\ell}(P)$ the sub level set

$$
V_{\ell}(P)=\left\{X \mid \max _{\alpha \in P}\left(\ell_{X}(\alpha)\right)<\ell\right\} .
$$

Then Bers's theorem guarantees that the sub level sets $V(P)=V_{L}(P)$ cover Teichmüller space.

Coarsely, the Weil-Petersson distance records the configuration of the sub level sets $V(P)$ with $\mathbf{P}(S)$ as its model. Indeed, if $P_{X}$ and $P_{Y}$ are pants decompositions for which $X \in V\left(P_{X}\right)$ and $Y \in V\left(P_{Y}\right)$, then the quasi-isometry of Theorem 1.1 arises from the comparability

$$
d_{\mathbf{P}}\left(P_{X}, P_{Y}\right) \asymp d_{\mathrm{WP}}(X, Y),
$$

where $d_{\mathbf{P}}\left(P_{X}, P_{Y}\right)$ is the minimum number of elementary moves required to travel from $P_{X}$ to $P_{Y}$ in $\mathbf{P}(S)$.

We briefly outline our proof of Theorem 1.1 and its application to Theorem 1.2

Outline of the proof of Theorem 1.1 Let $\mathbf{P}^{0}(S)$ denote the vertex set of $\mathbf{P}(S)$, and let $Q: \mathbf{P}^{0}(S) \rightarrow$ Teich $(S)$ be any map so that $Q(P) \in V(P)$. Applying work of S. Wolpert and H. Masur there is a uniform constant $D>0$ so that the Weil-Petersson diameter of $V(P)$ satisfies

$$
\operatorname{diam}_{W P}(V(P))<D
$$

for all $P \in \mathbf{P}^{0}(S)$. A simple argument shows that for any two pants decompositions $P_{1}$ and $P_{2}$ differing by an elementary move there is a single Riemann surface $X$ on which all curves in $P_{i}$ have length less than $L$. Thus $V\left(P_{1}\right) \cap V\left(P_{2}\right)$ is non-empty, and $Q$ is $2 D$-Lipschitz.

Given two pants decompositions $P_{1}$ and $P_{2}$ for which $V_{2 L}\left(P_{1}\right) \cap V_{2 L}\left(P_{2}\right) \neq \varnothing$, there is an $X \in V_{2 L}\left(P_{1}\right) \cap V_{2 L}\left(P_{2}\right)$ : i.e. a single Riemann surface on which each curve in $P_{1} \cup P_{2}$ has length at most $2 L$. It follows that there is a uniform $C>0$ so that the total intersection number satisfies

$$
i\left(P_{1}, P_{2}\right) \leq C,
$$

which in turn provides a uniform bound to the distance $d_{\mathbf{P}}\left(P_{1}, P_{2}\right)$ in $\mathbf{P}(S)$.

A compactness argument shows that each $X \in V(P)=V_{L}(P)$ lies a uniformly definite distance from $\partial V_{2 L}(P)$. Thus, a unit-length Weil-Petersson geodesic can 
always be covered by a uniform number of sub level sets $V_{2 L}(P)$. It follows that for any pair of pants decompositions $P$ and $P^{\prime}$ for which $V(P)$ and $V\left(P^{\prime}\right)$ contain the endpoints of a unit length Weil-Petersson geodesic, $P$ and $P^{\prime}$ have uniformly bounded distance in $\mathbf{P}(S)$. The theorem follows.

Outline of the proof of Theorem 1.2. The proof has two parts.

Bounding volume from below: The bound below of core volume in terms of the Weil-Petersson distance begins with an interpolation through the convex core

$$
h_{t}: Z_{t} \rightarrow \operatorname{core}(Q(X, Y))
$$

of 1-Lipschitz maps of hyperbolic surfaces. It follows that for each essential simple closed curve $\alpha$ we have

$$
\inf _{t} \ell_{Z_{t}}(\alpha) \geq \ell_{Q(X, Y)}(\alpha) .
$$

The path $Z_{t}$, then, only passes through sets $V(P)$ for which each element in $P$ has length less than $L$ in $Q(X, Y)$. Applying recent work of Masur and Minsky, we show that if a sequence $\left\{P_{1}, \ldots, P_{n}\right\}$ of pants decompositions is built from $N$ curves and makes bounded jumps, i.e.

$$
d_{\mathbf{P}}\left(P_{j}, P_{j+1}\right)<k,
$$

then its ends satisfy the bound $d_{\mathbf{P}}\left(P_{1}, P_{n}\right)<K_{0} N$, where $K_{0}$ depends only on $k$ and $S$.

The Margulis lemma forces closed geodesics with length less than $L$ in $Q(X, Y)$ that represent different homotopy classes to be uniformly equidistributed through the convex core. Since each such representative makes a definite contribution to core volume, the lower bound follows.

Bounding volume from above. Given pants decompositions $P_{X}$ and $P_{Y}$ so that $X \in V\left(P_{X}\right)$ and $Y \in V\left(P_{Y}\right)$, and a geodesic $G \subset \mathbf{P}(S)$ joining $P_{X}$ to $P_{Y}$, we consider the closed geodesics

$$
\operatorname{spin}(G)=\left\{\alpha^{*} \mid \alpha \in P \text { for } P \in G\right\},
$$

where $\alpha^{*}$ denotes the geodesic representative of $\alpha$ in $Q(X, Y)$. We build a straight triangulation $\mathcal{T}$ of all but a uniformly bounded volume portion of $\operatorname{core}(Q(X, Y))$ so that vertices of $\mathcal{T}$ lie on $\alpha^{*} \in \operatorname{spin}(G)$, the so-called spinning geodesics.

Our triangulation has the property that all but constant times $d_{\mathbf{P}}\left(P_{X}, P_{Y}\right)$ of the tetrahedra in $\mathcal{T}$ have at least one edge in a spinning geodesic $\alpha^{*}$. We then use a spinning trick: by homotoping the vertices around the geodesics in $\operatorname{spin}(G)$ keeping the triangulation straight, all tetrahedra with an edge in any $\alpha^{*}$ can be made to have arbitrarily small volume.

Since there is an a priori bound to the volume of a tetrahedron in $\mathbb{H}^{3}$, the remaining tetrahedra have uniformly bounded volume. The theorem then follows from the comparability $d_{\mathrm{WP}}(X, Y) \asymp d_{\mathbf{P}}\left(P_{X}, P_{Y}\right)$.

Geometrically finite hyperbolic 3-manifolds. We remark that simple generalizations of these techniques may be employed to obtain estimates for core volume of hyperbolic 3-manifolds that are not quasi-Fuchsian once the appropriate version of Weil-Petersson distance is defined. For example, given a hyperbolic 3-manifold $M_{\psi}$ that fibers over the circle with monodromy $\psi$, the volume of $M_{\psi}$ is comparable to the Weil-Petersson translation distance of $\psi$ (with constants depending only on the topology of the fiber). We take up these generalizations in [Br3]. 
Algebraic and geometric limits. As an application of Theorem 1.2, boundedness of the Weil-Petersson distance $d_{\mathrm{WP}}\left(X_{k}, Y_{k}\right)$ for sequences predicts the geometric finiteness of the geometric limit of $Q\left(X_{k}, Y_{k}\right)$.

The space $Q F(S)$ of all quasi-Fuchsian hyperbolic 3-manifolds lies in the space $A H(S)$ of all complete hyperbolic 3-manifolds $M$ marked by homotopy equivalences $(h: S \rightarrow M)$ so that $h_{*}$ sends peripheral elements of $\pi_{1}(S)$ to parabolic elements of $\pi_{1}(M)$. The space $A H(S)$ carries the algebraic topology or the compact-open topology on the induced representations $h_{*}: \pi_{1}(S) \rightarrow \operatorname{Isom}^{+}\left(\mathbb{H}^{3}\right)$ up to conjugacy.

In an algebraically convergent sequence $\left\{\left(h_{k}: S \rightarrow M_{k}\right)\right\}$ in $A H(S)$, normalizing the induced representations $\rho_{k}=\left(h_{k}\right)_{*}$ to converge on generators one may always extract a subsequence so that the groups $\rho_{k}\left(\pi_{1}(S)\right)=\Gamma_{k}$ converge in the GromovHausdorff topology on discrete subgroups of $\operatorname{Isom}^{+}\left(\mathbb{H}^{3}\right)$, or geometrically, to a limit $\Gamma_{G}$. A central issue in the deformation theory of hyperbolic 3-manifolds is to understand the geometric limit $N_{G}=\mathbb{H}^{3} / \Gamma_{G}$.

Applying Theorem 1.2 we obtain the following criterion:

Theorem 1.4. Let $Q\left(X_{k}, Y_{k}\right) \rightarrow Q_{\infty}$ be an algebraically convergent sequence in $A H(S)$ with geometric limit $N_{G}$. Then $N_{G}$ is geometrically finite if and only if there is a $K>0$ for which

$$
d_{\mathrm{WP}}\left(X_{k}, Y_{k}\right)<K
$$

for all $k$.

Note that geometric finiteness of $N_{G}$ implies geometric finiteness of $Q_{\infty}$ but not conversely.

History and references. The fundamental properties of the Weil-Petersson metric we use are discussed in WWol1], Wol3], [Wol4], and [Mas. The pants graph is the 1-skeleton of the pants complex, introduced in [HT] (see also [HLS]), which is there proven to be connected. The relation of the pants graph to the Weil-Petersson metric is similar in spirit to the relative hyperbolicity theorem for Teichmüller space of [MM1, where the (related) complex of curves is shown to be quasi-isometric to the electric Teichmüller space, and to be Gromov-hyperbolic (the pants complex and the Weil-Petersson metric are not in general Gromov-hyperbolic $[\overline{\mathrm{BF}}]$ ). For more on quasi-Fuchsian manifolds and their algebraic and geometric limits, see Th1], [Brs1, [Mc2, Mc1, [Br2, and [Ot].

Plan of the paper. After discussing the fundamental work of S. Wolpert and H. Masur on the Weil-Petersson metric that will serve as our jumping off point in Section 2 we prove the comparability of Weil-Petersson distance and pants distance (Theorem 1.1) in Section 3. We then establish the lower bound on $\operatorname{vol}(X, Y)$ in terms of the distance $d_{\mathbf{P}}\left(P_{X}, P_{Y}\right)$ in Section 4 Section 5 applies the combinatorics of pants decompositions along a geodesic $G \subset \mathbf{P}(S)$ joining $P_{X}$ to $P_{Y}$ to bound volume from above in terms of pants distance. Theorem 1.2 then follows from the comparability of Theorem 1.1. We conclude with applications to the study of geometric limits, proving Theorem 1.4 .

\section{The extended Weil-Petersson metric}

Let $S$ be a compact oriented surface of negative Euler characteristic. We allow $S$ to have boundary and let $\operatorname{int}(S)$ denote its interior. Let $\mathcal{S}$ denote the set of isotopy classes of essential, non-peripheral, simple closed curves on $S$. 
A pants decomposition $P \subset \mathcal{S}$ is a maximal collection of isotopy classes with pairwise disjoint representatives on $S$. The usual geometric intersection number $i(\alpha, \beta)$ of a pair $(\alpha, \beta) \in \mathcal{S} \times \mathcal{S}$ generalizes to a total intersection number $i\left(P, P^{\prime}\right)$ of pants decompositions by summing the geometric intersections of their components.

The Teichmüller space Teich $(S)$ of $S$ parameterizes finite area hyperbolic structures on $S$ up to isotopy. Points in $\operatorname{Teich}(S)$ are pairs $(f, X)$, where $X$ is a finite area hyperbolic surface $X$ equipped with a homeomorphism $f: \operatorname{int}(S) \rightarrow X$, up to the equivalence $(f, X) \sim(g, Y)$ if there is an isometry $\phi: X \rightarrow Y$ for which $\phi \circ f \simeq g$. A pants decomposition $P=\alpha_{1} \cup \ldots \cup \alpha_{|P|}$ determines Fenchel-Nielsen coordinates

$$
\left(\ell_{X}\left(\alpha_{1}\right), \ldots, \ell_{X}\left(\alpha_{|P|}\right), \theta_{X}\left(\alpha_{1}\right), \ldots, \theta_{X}\left(\alpha_{|P|}\right)\right) \in \mathbb{R}_{+}^{|P|} \times \mathbb{R}^{|P|}
$$

for each $X \in \operatorname{Teich}(S)$, indicating $X$ is assembled from hyperbolic pairs of pants with boundary lengths prescribed by $\ell_{X}\left(\alpha_{i}\right)$ glued together twisted by $\theta_{X}\left(\alpha_{i}\right)$. (For more on Teichmüller space and Fenchel-Nielsen coordinates see [IT] or Gard].)

The Weil-Petersson metric. Each $X \in \operatorname{Teich}(S)$ is naturally a complex 1manifold via its uniformization $X=\mathbb{H}^{2} / \Gamma$ as the quotient of the upper half-plane by a Fuchsian group. The Teichmüller space has a complex manifold structure of dimension $3 g-3+n$, where $S$ has genus $g$ and $n$ boundary components.

The space of holomorphic quadratic differentials $Q(X)$ on $X \in \operatorname{Teich}(S)$ (holomorphic forms of type $\phi(z) d z^{2}$ on $\left.X\right)$ is naturally the cotangent space $T_{X}^{*} \operatorname{Teich}(S)$ to $\operatorname{Teich}(S)$ at $X$. The Weil-Petersson metric on Teich $(S)$ unifies the hyperbolic and holomorphic perspectives on $X$ : it arises from the $L^{2}$ inner product

$$
\langle\varphi, \psi\rangle_{\mathrm{WP}}=\frac{i}{2} \int_{X} \frac{\varphi \bar{\psi}}{\rho^{2}}
$$

on $Q(X)$, where $\rho(z)|d z|$ is the hyperbolic metric on $X$, by the usual pairing

$$
(\mu, \varphi)_{X}=\int_{X} \mu \varphi
$$

between $T_{X} \operatorname{Teich}(S)$ and $T_{X}^{*} \operatorname{Teich}(S)$ (see, e.g., [Wol3, Sec. 1]). In what follows, we will be interested only in the Riemannian part $g_{\mathrm{WP}}$ of the Weil-Petersson metric, and its associated distance function $d_{\mathrm{WP}}(.,$.$) on Teich (S)$.

The Weil-Petersson metric has negative sectional curvature [Tro], Wol2], and the modular group $\operatorname{Mod}(S)$ (the group of isotopy classes of orientation preserving homeomorphisms of $S$ ) acts by isometries of $g_{\mathrm{WP}}$. Thus, $g_{\mathrm{WP}}$ descends to a metric on the moduli space $\mathcal{M}(S)=\operatorname{Teich}(S) / \operatorname{Mod}(S)$.

Work of S. Wolpert shows two important properties of the Weil-Petersson metric we will use:

WPI: The Weil-Petersson metric is not complete: "pinching geodesics" in the Teichmüller metric (which leave every compact set of Teich $(S)$ ) have finite WeilPetersson length [Wol1].

WPII: The Weil-Petersson metric is geodesically convex: in fact, for $\alpha \in \mathcal{S}$ the length function $\ell_{(.)}(\alpha)$ is strictly convex along Weil-Petersson geodesics [Wol3].

The augmented Teichmüller space. In $[\mathrm{Mas}, \mathrm{H}$. Masur shows that the WeilPetersson metric extends to the augmented Teichmüller space $\overline{\text { Teich }(S)}$ obtained by adding boundary Teichmüller spaces consisting of marked noded Riemann surfaces, which we now describe (see [Brs3] for a detailed discussion). 
A Riemann surface with nodes $W$ is a connected complex space so that each point $p \in W$ has a neighborhood isomorphic to $\{z \in \mathbb{C}|| z \mid<1\}$ or isomorphic to $\left\{(z, w) \in \mathbb{C}^{2}|| z|<1| w \mid,<1\right.$, and $\left.z w=0\right\}$ by an isomorphism sending $p$ to $(0,0) \in \mathbb{C}^{2}$. In the latter case, $p$ is called a node of $X$. The complement of the nodes is a union of Riemann surfaces called the pieces of $W$. We say $W$ is hyperbolic if each piece of $W$ admits a complete finite-area hyperbolic structure.

Given curves $\nu_{1}, \ldots, \nu_{j}$ in a pants decomposition $P=\left\{\alpha_{1}, \ldots, \alpha_{|P|}\right\}$ of $S$, a marked noded hyperbolic surface pinched along $\nu_{1}, \ldots, \nu_{j}$ is a noded hyperbolic Riemann surface $W$ together with a continuous map

$$
f: \operatorname{int}(S) \rightarrow W
$$

so that $\left.f\right|_{S-\nu_{1} \cup \ldots \cup \nu_{j}}$ is a homeomorphism to the union of the pieces of $W$. Let $S-\mathcal{N}\left(\nu_{1}\right) \cup \ldots \cup \mathcal{N}\left(\nu_{j}\right)=S_{1} \cup \ldots \cup S_{k}$, where $\mathcal{N}\left(\nu_{i}\right)$ are pairwise disjoint open collars about each $\nu_{i}$. Then the pair $(f, W)$ determines a point

$$
\operatorname{Teich}\left(S_{1}\right) \times \ldots \times \operatorname{Teich}\left(S_{k}\right)
$$

in the product Teichmüller space by taking the restriction of $f$ to each component of $\operatorname{int}(S)-\nu_{1} \cup \ldots \cup \nu_{j}$ as a marking on each piece of $W$. A marked piece of $W_{l} \in \operatorname{Teich}\left(S_{l}\right), 1 \leq l \leq k$, has Fenchel-Nielsen coordinates with respect to the elements of the pants decomposition $P$ that lie in $S_{l}$.

Two marked hyperbolic noded surfaces $\left(f_{1}, W_{1}\right)$ and $\left(f_{2}, W_{2}\right)$ are equivalent, if there is continuous map $\phi: W_{1} \rightarrow W_{2}$ that is isometric on each piece of $W_{1}$ for which $\phi \circ f_{1}=f_{2}$ after precomposition with an isotopy of $S$.

The augmented Teichmüller space $\overline{\text { Teich }(S)}$ is obtained by adjoining equivalence classes of marked noded hyperbolic surfaces to Teich $(S)$. The topology on $\overline{\operatorname{Teich}(S)}$ is given as follows. Given a pants decomposition $P$, and a point $W \in \overline{\operatorname{Teich}(S)}$ with curves $\nu_{1} \cup \ldots \cup \nu_{j}$ in $P$ pinched to nodes, we extend the Fenchel-Nielsen coordinates to $W$ by defining the coordinates $\ell_{W}\left(\nu_{i}\right)=0$. Then a neighborhood of $W$ in $\overline{\text { Teich }(S)}$ consists of (possibly noded) hyperbolic Riemann surfaces $X$ whose length coordinates $\ell_{X}\left(\alpha_{p}\right)$ are close to those of $W$ for $p=1, \ldots, 3 g-3+n$, and whose twist coordinates $\theta_{X}\left(\alpha_{p}\right)$ are close to those of $X$ for each $p$ such that $\alpha_{p} \neq \nu_{i}$ (see [IT, App. B]).

The Weil-Petersson metric extends to the augmented Teichmüller space as its completion (see $[\mathrm{Mas}]$ ), giving a $\operatorname{Mod}(S)$ invariant metric on $\overline{\operatorname{Teich}(S)}$. The quotient

$$
\overline{\operatorname{Teich}(S)} / \operatorname{Mod}(S)=\overline{\mathcal{M}(S)},
$$

the familiar Mayer-Mumford-Deligne compactification of the moduli space (see [Brs3]), inherits a complete extension of the Weil-Petersson metric on $\mathcal{M}(S)$. We denote the corresponding distance by

$$
d_{\overline{\mathrm{WP}}}: \overline{\text { Teich }(S)} \times \overline{\text { Teich }(S)} \rightarrow \mathbb{R}_{\geq 0} .
$$

Evidently, the failure of completeness of the Weil-Petersson metric occurs at limits of pinching sequences $X_{t}$ for which the length coordinates $\ell_{X_{t}}\left(\nu_{i}\right)$ tend to zero for some collection of curves in a pants decomposition $P$.

Given a pants decomposition $P$ and a collection $\alpha_{1}, \ldots, \alpha_{k}$ of curves in $P$, the minimal distance from a point $X \in \operatorname{Teich}(S)$ to a noded Riemann surface $Z$ with nodes along $\alpha_{1}, \ldots, \alpha_{k}$ is estimated in terms of the geodesic length sum

$$
\ell=\ell_{X}\left(\alpha_{1}\right)+\ldots+\ell_{X}\left(\alpha_{k}\right)
$$


of the lengths of $\alpha_{i}$ on $X$ by

$$
d \overline{\mathrm{WP}}(X, Z)=\sqrt{2 \pi \ell}+O\left(\ell^{2}\right)
$$

(see [Wol5, Cor. 21]).

Remark. This estimate is a recent improvement of similar estimates originally obtained in Wol4 Ex. 4.3] and cited in earlier versions of this manuscript.

Sub level sets. We recall the following theorem of Bers.

Theorem 2.1 (Bers). There is a constant $L>0$ depending only on $S$ such that for any $X \in \operatorname{Teich}(S)$ there is a pants decomposition $P$ such that $\ell_{X}(\alpha)<L$ for each $\alpha \in P$.

We call this $L$ the Bers constant for $S$.

Given a pants decomposition $P$, and a positive real number $\ell \in \mathbb{R}_{+}$, we consider the sub level set

$$
V_{\ell}(P)=\left\{X \in \operatorname{Teich}(S) \mid \max _{\alpha \in P}\left\{\ell_{X}(\alpha)\right\}<\ell\right\} .
$$

Then by Bers's theorem, the union of the sets $V_{L}(P)$ over all pants decompositions gives an open cover of Teich $(S)$. Because $L$ depends only on $S$, we abbreviate

$$
V(P)=V_{L}(P) .
$$

Then we have the following:

Proposition 2.2. The sub level sets $V(P)$ have the following properties.

(1) Each $V(P)$ is convex in the Weil-Petersson metric, and

(2) there is a constant $D>0$, depending only on $S$, for which the WeilPetersson diameter $\operatorname{diam}_{\mathrm{WP}}(V(P))$ is less than $D$.

Proof. Geodesic convexity of $V(P)$ follows immediately from WPII, the convexity of the geodesic length functions $\ell_{X}().(\alpha)$ for each $\alpha \in P$.

To see that each $V(P)$ has bounded Weil-Petersson diameter, let $W_{P}$ be the (unique) maximally noded Riemann surface where each curve in $P$ is pinched. By equation (2.1) there is a constant $C(L)$ so that for each $X \in V(P)$ we have

$$
d_{\overline{\mathrm{WP}}}\left(X, W_{P}\right)<C(L) \text {. }
$$

By the triangle inequality for $d_{\overline{\mathrm{WP}}}$, if $X$ and $Y$ lie in $V(P)$, then the distance $d_{\overline{\mathrm{WP}}}(X, Y)$ is bounded by $2 C(L)$. By geodesic convexity of $V(P)$ the geodesic joining $X$ to $Y$ lies in $V(P)$, so we have the bound

$$
d_{\mathrm{WP}}(X, Y)<2 C(L)
$$

on $d_{\mathrm{WP}}(X, Y)$, which we set equal to $D$.

\section{A combinatorial Weil-Petersson distance}

In this section, we relate the coarse geometry of the Weil-Petersson metric to the pants graph $\mathbf{P}(S)$ defined in the introduction. We do this by exhibiting a quasi-isometry between the two spaces with their respective distances. 
Definition 3.1. Given $k_{1}>1$ and $k_{2}>0$, a map $f:(X, d) \rightarrow\left(Y, d^{\prime}\right)$ of metric spaces is a $\left(k_{1}, k_{2}\right)$-quasi-isometric embedding if for each pair of points $x$ and $y$ in $X$ we have

$$
\frac{d(x, y)}{k_{1}}-k_{2} \leq d^{\prime}(f(x), f(y)) \leq k_{1} d(x, y)+k_{2} .
$$

The spaces $(X, d)$ and $\left(Y, d^{\prime}\right)$ are quasi-isometric if for some $k_{1}>1$ and $k_{2}>0$ there are $\left(k_{1}, k_{2}\right)$-quasi-isometric embeddings from $(X, d)$ to $\left(Y, d^{\prime}\right)$ and from $\left(Y, d^{\prime}\right)$ to $(X, d)$. In practice, it suffices to exhibit a quasi-isometry from $(X, d)$ to $\left(Y, d^{\prime}\right)$, namely, a quasi-isometric embedding with uniformly dense image. Given such a quasi-isometry from $(X, d)$ to $\left(Y, d^{\prime}\right)$, a quasi-isometric embedding from $\left(Y, d^{\prime}\right)$ to $(X, d)$ is readily constructed, so the spaces are quasi-isometric.

Let

$$
Q: \mathbf{P}^{0}(S) \rightarrow \operatorname{Teich}(S)
$$

be any embedding of the vertices $\mathbf{P}^{0}(S)$ of $\mathbf{P}(S)$ into Teich $(S)$ so that $Q(P)$ lies in $V(P)$. The main theorem of this section is the following:

Theorem 3.2. The map $Q$ is a quasi-isometry of the 0 -skeleton $\mathbf{P}^{0}(S)$ of $\mathbf{P}(S)$ with $\operatorname{Teich}(S)$ with its Weil-Petersson distance.

Proof. By the uniform bound

$$
\operatorname{diam}_{W P}(V(P))<D
$$

on the diameter of $V(P)$, the image $Q(\mathbf{P}(S))$ is $D$-dense in Teich $(S)$. It suffices, then, to show that there are uniform constants $A_{1} \geq 1$ and $A_{2} \geq 0$ so that

$$
\frac{1}{A_{1}} d_{\mathbf{P}}\left(P_{0}, P_{1}\right)-A_{2} \leq d_{\mathrm{WP}}\left(Q\left(P_{0}\right), Q\left(P_{1}\right)\right) \leq A_{1} d_{\mathbf{P}}\left(P_{0}, P_{1}\right)+A_{2} .
$$

We first show that the map $Q$ is $2 D$-Lipschitz. Given $P_{0}$ and $P_{1}$ such that

$$
d_{\mathbf{P}}\left(P_{0}, P_{1}\right)=1,
$$

$P_{0}$ and $P_{1}$ differ by a single elementary move. Let $\alpha \in P_{0}$ and $\beta \in P_{1}$ be the curves involved in this elementary move, i.e. $P_{0}-\alpha=P_{1}-\beta$ and $i(\alpha, \beta)=1$ or 2 depending on whether the component $S_{\alpha} \subset S-\left(P_{0}-\alpha\right)$ containing $\alpha$ is a punctured torus or four-times punctured sphere.

Let $Z \in \operatorname{Teich}\left(S_{\alpha}\right)$ be the "square" punctured torus: i.e. $Z$ is obtained by identifying opposite sides of an ideal square in $\mathbb{H}^{2}$ with order-4 rotational symmetry about the origin in the disk model of $\mathbb{H}^{2}$.

Marking $Z$ so that the common perpendiculars to the opposite sides descend to closed geodesics $\alpha$ and $\beta$ on $Z$ (see Figure 4 ), we have by symmetry that the length $\ell_{Z}(\alpha)$ equals the length $\ell_{Z}(\beta)$, which is the shortest length of any non-peripheral simple closed curve on $Z$. In particular, we have

$$
\ell_{Z}(\alpha)<L\left(S_{\alpha}\right)
$$

where $L\left(S_{\alpha}\right)$ is the Bers constant for $S_{\alpha}$.

Let $(f, W) \in \overline{\text { Teich }(S)}$ be the noded Riemann surface with nodes at each $\gamma \in$ $P-\alpha$, and one non-rigid piece $\left.f\right|_{S_{\alpha}} \rightarrow Z$. For any $W^{\prime} \in \operatorname{Teich}(S)$ sufficiently close to $W$, if $W^{\prime} \in V\left(P^{\prime}\right)$, then $P^{\prime}$ contains an essential non-peripheral curve in $S_{\alpha}$. Taking $W^{\prime}$ arbitrarily close to $W$, we may conclude that $L\left(S_{\alpha}\right) \leq L(S)=L$. Then for any Riemann surface $W^{\prime} \in \operatorname{Teich}(S)$ sufficiently close to $W \in \overline{\operatorname{Teich}(S)}$ we have

$$
\ell_{W^{\prime}}(\gamma)<L
$$




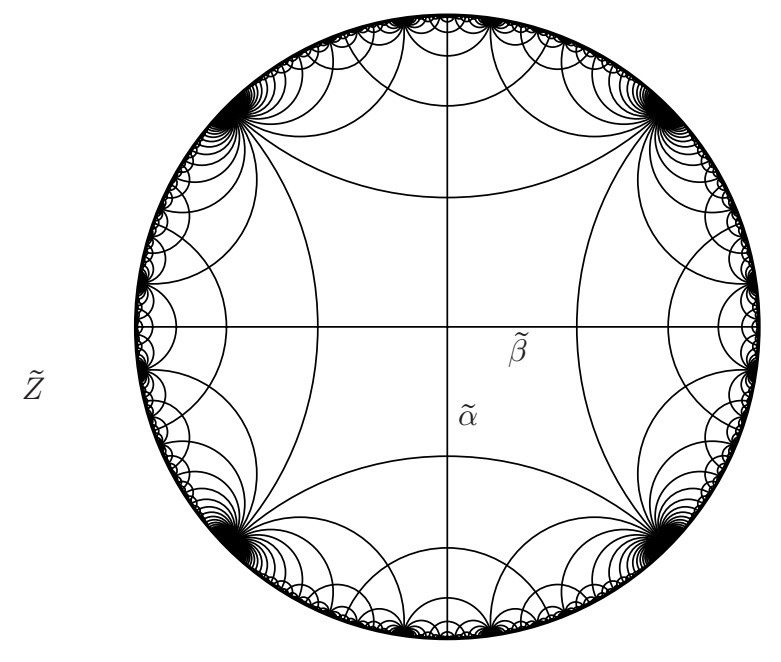

Figure 4 . The cover $\widetilde{Z}$ with lifts of $\alpha$ and $\beta$ passing through the origin.

for each $\gamma \in P_{0} \cup P_{1}$. In other words, $W^{\prime}$ lies in the intersection $V\left(P_{0}\right) \cap V\left(P_{1}\right)$. Letting $Z$ be the double of the symmetric ideal square described above, a similar argument handles the genus- 0 case.

Therefore we may conclude that

$$
d_{\mathrm{WP}}\left(Q\left(P_{0}\right), Q\left(P_{1}\right)\right)<2 D \quad \text { when } \quad d_{\mathbf{P}}\left(P_{0}, P_{1}\right)=1,
$$

so by the triangle inequality, $Q$ is $2 D$-Lipschitz. To show that for some $A_{1}$ and $A_{2}$ the inequality

$$
\frac{1}{A_{1}} d_{\mathbf{P}}\left(P_{0}, P_{1}\right)-A_{2} \leq d_{\mathrm{WP}}\left(Q\left(P_{0}\right), Q\left(P_{1}\right)\right)
$$

holds is somewhat more delicate. We break this into a series of lemmas.

Lemma 3.3. Given $L^{\prime}>L$, there is an integer $B>0$ so that given $P$ and $P^{\prime}$ in $\mathbf{P}(S)$ for which $V_{L^{\prime}}(P) \cap V_{L^{\prime}}\left(P^{\prime}\right) \neq \varnothing$, we have $d_{\mathbf{P}}\left(P, P^{\prime}\right) \leq B$.

Proof. The hypotheses imply that there is some $X \in \operatorname{Teich}(S)$ so that $\ell_{X}(\alpha)<L^{\prime}$ for each $\alpha \in P \cup P^{\prime}$. By an application of the collar lemma [BuS, Thm. 4.4.6] there is a constant $C$ depending only $L^{\prime}$ and $S$ so that the total geometric intersection number $i\left(P, P^{\prime}\right)$ satisfies

$$
i\left(P, P^{\prime}\right) \leq C
$$

Let $\mathbf{T w}(P) \cong \mathbb{Z}^{|P|}$ denote the subgroup of $\operatorname{Mod}(S)$ generated by Dehn twists about the curves in $P$. Then the function $i(P,):. \mathbf{P}^{0}(S) \rightarrow \mathbb{Z}$ descends to a function

$$
i(P, .): \mathbf{P}^{0}(S) / \mathbf{T w}(P) \rightarrow \mathbb{Z}
$$

whose sub level sets are bounded: in other words, there are only finitely many equivalence classes

$$
\left\{\left[P_{1}\right], \ldots,\left[P_{c}\right]\right\} \subset \mathbf{P}^{0}(S) / \mathbf{T w}(P)
$$

for which $i\left(P,\left[P_{j}\right]\right) \leq C$.

Since $d_{\mathbf{P}}(P,$.$) also descends to a function$

$$
d_{\mathbf{P}}(P, .): \mathbf{P}^{0}(S) / \mathbf{T w}(P) \rightarrow \mathbb{Z}
$$


on $\mathbf{P}^{0}(S) / \mathbf{T w}(P)$, we have

$$
d_{\mathbf{P}}\left(P, P^{\prime}\right) \leq B
$$

where

$$
B=\max _{j=1, \ldots, c}\left\{d_{\mathbf{P}}\left(P,\left[P_{j}\right]\right)\right\}
$$

Lemma 3.4. Given $L^{\prime}>L$, there is an integer $J>0$, so that if $X_{t}, t \in[0,1]$, is a unit-length Weil-Petersson geodesic joining $X_{0}$ and $X_{1}$, then there exist pants decompositions $P_{1}, \ldots, P_{J}$ so that $\left\{X_{t}\right\}_{t=0}^{1}$ lies in the union

$$
V_{L^{\prime}}\left(P_{1}\right) \cup \ldots \cup V_{L^{\prime}}\left(P_{J}\right) \text {. }
$$

Proof. Recall from Theorem 2.1 that the sets $V(P) \subset V_{L^{\prime}}(P)$ cover Teich $(S)$. Let $P_{1}, \ldots, P_{m}$ determine sets $V\left(P_{1}\right), \ldots, V\left(P_{m}\right)$ so that for each $t \in[0,1]$ we have

$$
X_{t} \in V\left(P_{1}\right) \cup \ldots \cup V\left(P_{m}\right) \text {. }
$$

Let

be the function

$$
d_{L^{\prime}, P}: \partial V(P) \rightarrow \mathbb{R}_{+}
$$

$$
d_{L^{\prime}, P}(X)=\inf _{Y \in \partial V_{L^{\prime}}(P)} d_{\mathrm{WP}}(X, Y) .
$$

We claim that there is an $\epsilon_{0}$ depending only on $L$, and $L^{\prime}$ so that

$$
d_{L^{\prime}, P}(X)>\epsilon_{0} .
$$

The function $d_{L^{\prime}, P}(X)$ naturally extends to the metric completion $\overline{\partial V(P)}$ of $\partial V(P)$, and $d_{L^{\prime}, P}(X)$ is invariant under the action of $\mathbf{T} \mathbf{w}(P)$.

Let $\left\{\left(\ell_{i}, \theta_{i}\right) \in \mathbb{R}_{+}^{|P|} \times \mathbb{R}^{|P|}\right\}$ denote Fenchel-Nielsen coordinates for $\operatorname{Teich}(S)$ adapted to the pants decomposition $P$. To extend these Fenchel Nielsen coordinates to the completion, we denote by

$$
\mathbb{R}_{\geq 0} \times \mathbb{R} / \sim
$$

the quotient of $\mathbb{R}_{\geq 0} \times \mathbb{R}$ by the equivalence relation $(0, \theta) \sim\left(0, \theta^{\prime}\right)$. Then the completion $\overline{V_{L^{\prime}}(P)}$ of $V_{L^{\prime}}(P)$ in Teich $(S)$ admits extended Fenchel-Nielsen coordinates

$$
\overline{V_{L^{\prime}}(P)}=\left\{\left(\ell_{i}, \theta_{i}\right) \in \mathbb{R}^{+} \times \mathbb{R} / \sim\left|\ell_{i} \leq L^{\prime}, i=1, \ldots,\right| P \mid\right\},
$$

where each point with $\ell_{j}=0$ for some $j$ lies in the completion. The extended isometric action of $\mathbf{T w}(P)$ on $\overline{\operatorname{Teich}(S)}$ is cocompact on $\overline{V_{L^{\prime}}(P)}$, since $\mathbf{T w}(P)$ preserves each length coordinate and acts by translations on each twist coordinate.

In these extended Fenchel-Nielsen coordinates, the completion $\overline{\partial V_{L^{\prime}}(P)}$ of the boundary $\partial V_{L^{\prime}}(P)$ is the locus of coordinates for which $\ell_{j}=L^{\prime}$ for some $j \in$ $1, \ldots,|P|$. Thus

$$
\overline{\partial V_{L^{\prime}}(S)} / \mathbf{T w}(P)
$$

is a closed subset of the compact set $\overline{V_{L^{\prime}}(P)} / \mathbf{T w}(P)$ and is thus compact.

Since the quotients

$$
\overline{\partial V_{L^{\prime}}(P)} / \mathbf{T w}(P) \text { and } \overline{\partial V(P)} / \mathbf{T w}(P)
$$

are disjoint compact subsets of $\overline{V_{L^{\prime}}(P)} / \mathbf{T w}(P)$, it follows that the function

$$
\mathcal{I}_{L^{\prime}}: \mathbf{P}^{0}(S) \rightarrow \mathbb{R}_{+}
$$


given by

$$
\mathcal{I}_{L^{\prime}}(P)=\inf _{X \in \partial V(P)} d_{L^{\prime}, P}(X)
$$

is positive. But $\mathcal{I}_{L^{\prime}}$ is $\operatorname{Mod}(S)$-invariant, so it descends to a function

$$
\mathcal{I}_{L^{\prime}}: \mathbf{P}^{0}(S) / \operatorname{Mod}(S) \rightarrow \mathbb{R}_{+} .
$$

Since $\mathbf{P}^{0}(S) / \operatorname{Mod}(S)$ is finite, we may set $\epsilon_{0}$ equal to the infimum of $\mathcal{I}_{L^{\prime}}([P])$ over the finite number of equivalence classes $[P] \in \mathbf{P}^{0}(S) / \operatorname{Mod}(S)$.

If $X_{t_{0}}$ lies in $V(P)$, then $X_{t}$ lies in $V_{L^{\prime}}(P)$, provided $t$ lies in $\left(t_{0}-\epsilon_{0}, t_{0}+\epsilon_{0}\right)$. It follows that after setting $J$ equal to the least integer greater than $2 / \epsilon_{0}$, we may select from the pants decompositions $P_{1}, \ldots, P_{m}$ pants decompositions $P_{1}, \ldots, P_{J}$ (possibly with repetition) so that

$$
X_{t} \in V_{L^{\prime}}\left(P_{1}\right) \cup \ldots \cup V_{L^{\prime}}\left(P_{J}\right)
$$

for each $t \in[0,1]$.

Continuation of the proof of Theorem 3.2 To complete the proof of Theorem 3.2 let $X_{t}$ be the Weil-Petersson geodesic joining arbitrary distinct Riemann surfaces $X$ and $Y$ in Teich $(S)$. Let $P_{X}$ and $P_{Y}$ be pants decompositions for which $X$ lies in $V\left(P_{X}\right)$ and $Y$ lies in $V\left(P_{Y}\right)$. Let $I(P) \subset[0,1]$ denote the values of $t$ for which $X_{t} \in V_{2 L}(P)$. By convexity of $V(P)$ (Proposition 2.2) each $I(P)$ is an interval.

Taking $L^{\prime}=2 L$, Lemma 3.4 provides a $J>0$ and a sequence $\left\{P_{j}\right\}_{j=0}^{N} \in \mathbf{P}(S)$ so that

- $X(t)$ is covered by the union $\bigcup_{j} I\left(P_{j}\right)$,

- the least upper bound of $I\left(P_{j}\right)$ lies in $I\left(P_{j+1}\right)$, and

- $N \leq J\left(d_{\mathrm{WP}}(X, Y)+1\right)$.

Thus, we have

$$
\frac{N}{J}-1 \leq d_{\mathrm{WP}}(X, Y) .
$$

Moreover, for successive pants decompositions $P_{j}, P_{j+1}$, we have

$$
V_{2 L}\left(P_{j}\right) \cap V_{2 L}\left(P_{j+1}\right) \neq \varnothing,
$$

so applying Lemma 3.3 with $L^{\prime}=2 L$, we have a $B>0$ for which

$$
d_{\mathbf{P}}\left(P_{X}, P_{Y}\right) \leq B N .
$$

Combining equations 3.2 and 3.3 , we have

$$
\frac{d_{\mathbf{P}}\left(P_{X}, P_{Y}\right)}{B J}-1 \leq d_{\mathrm{WP}}\left(Q\left(P_{X}\right), Q\left(P_{Y}\right)\right),
$$

where $B$ and $J$ depend only on $L$, which depends only on $S$. Setting $A_{1}=B J$ and $A_{2}=1$ concludes the proof of Theorem 3.2 .

\section{Bounding THE CORE VOlume From Below}

To simplify notation, let

$$
C(X, Y)=\operatorname{core}(Q(X, Y))
$$

and recall that $\operatorname{vol}(X, Y)$ denotes the convex core volume $\operatorname{vol}(\operatorname{core}(Q(X, Y)))$. In this section we prove 
Theorem 4.1. Given $S$, there are constants $K_{1}>1$ and $K_{2}>0$ so that

$$
\frac{1}{K_{1}} d_{\mathrm{WP}}(X, Y)-K_{2} \leq \operatorname{vol}(X, Y) .
$$

The proof is given as a series of lemmas.

Fix attention on a given quasi-Fuchsian manifold $Q(X, Y)$. Given a constant $L_{0}>0$, let $\mathcal{S}_{<L_{0}} \subset \mathcal{S}$ denote the set of isotopy classes

$$
\mathcal{S}_{<L_{0}}=\left\{\alpha \in \mathcal{S} \mid \ell_{Q(X, Y)}(\alpha)<L_{0}\right\} .
$$

Theorem 4.1 will follow from a linear lower bound on $\operatorname{vol}(X, Y)$ given in terms of the size of $\mathcal{S}_{<L}$ (Lemma 4.8) and the following lemma.

Lemma 4.2. Let $P_{X}$ and $P_{Y}$ be pants decompositions so that $X \in V\left(P_{X}\right)$ and $Y \in V\left(P_{Y}\right)$. Then there is a constant $K$ depending only on $S$ so that

$$
d_{\mathbf{P}}\left(P_{X}, P_{Y}\right) \leq K\left|\mathcal{S}_{<L}\right| .
$$

The lemma will follow from the following general result on paths in $\mathbf{P}(S)$ that are built out of a given collection of curves in $\mathcal{S}$. For reference, let

$$
\pi_{\mathcal{S}}: \mathbf{P}^{0}(S) \rightarrow \mathcal{S}
$$

denote the set-valued projection that assigns to each $P$ the collection of curves used to build it.

Lemma 4.3. Let $k \in \mathbb{N}$ and let $g=\left\{P_{I}=P_{0}, \ldots, P_{N}=P_{T}\right\} \subset \mathbf{P}(S)$ be a sequence of pants decompositions with the property that $d_{\mathbf{P}}\left(P_{j}, P_{j+1}\right)<k$. Let $\mathcal{S}_{g} \subset \mathcal{S}$ denote the image of $g$ under the projection $\pi_{\mathcal{S}}$. There is a constant $K_{0}>0$ depending on $k$ and $S$ so that

$$
d_{\mathbf{P}}\left(P_{I}, P_{T}\right)<K_{0}\left|\mathcal{S}_{g}\right| .
$$

The complex of curves. The graph $\mathbf{P}(S)$ is related to the complex of curves $\mathcal{C}(S)$, introduced by W. Harvey $\mathrm{Har}$. To prove Lemma 4.3 we describe recent work of Masur and Minsky on $\mathcal{C}(S)$. The main result of [MM1] shows that $\mathcal{C}(S)$ is in fact a Gromov hyperbolic metric space with the metric obtained by making each simplex a standard Euclidean simplex. Its sequel [MM2] introduces a theory of hierarchies of so-called "tight geodesics" in $\mathcal{C}(S)$ and in subcomplexes $\mathcal{C}(Y)$ for essential subsurfaces $Y \subset S$. Such hierarchies and their hyperbolicity properties play an integral role in our control of volume.

To describe the topological type of $S$, we let

$$
d(S)=\operatorname{dim}_{\mathbb{C}}(\operatorname{Teich}(S))=3 g-3+n,
$$

where $S$ has genus $g$ with $n$ boundary components. We consider only those surfaces $S$ for which $\operatorname{int}(S)$ admits a hyperbolic structure (so $d(S)>0)$.

The complex of curves $\mathcal{C}(S)$ is a simplicial complex with 0-skeleton $\mathcal{S}$, and higherdimensional simplices described as follows:

- for $d(S)>1$ and $k \geq 1, k$-simplices of $\mathcal{C}(S)$ span $k+1$-tuples $\alpha_{1}, \ldots, \alpha_{k+1}$ of vertices for which $i\left(\alpha_{i}, \alpha_{j}\right)=0$, and

- if $d(S)=1, \mathcal{C}(S)$ is a 1-complex whose edges join vertices $\alpha$ and $\alpha^{\prime}$ in $\mathcal{C}(S)$ that intersect minimally; i.e. $\mathcal{C}(S)=\mathbf{P}(S)$. 
Given an essential subsurface $Y \subset S$ with $d(Y) \geq 2$, the curve complex $\mathcal{C}(Y)$ is naturally a subcomplex of $\mathcal{C}(S)$. Given a set $W$ let $\mathcal{P}(W)$ denote its power set, i.e. the set of all subsets of $W$. Masur and Minsky define a projection

$$
\pi_{Y}: \mathcal{C}(S) \rightarrow \mathcal{P}(\mathcal{C}(Y))
$$

by setting $\pi(\alpha)=\alpha$ if $\alpha \in \mathcal{C}(Y)$, and taking

$$
\pi_{Y}(\alpha)=\bigcup_{\alpha^{\prime} \subset \alpha \cap Y} \partial \mathcal{N}\left(\alpha^{\prime} \cup \partial_{\alpha^{\prime}} Y\right)
$$

where $\alpha^{\prime}$ is an arc of essential intersection of $\alpha$ with $Y, \partial_{\alpha^{\prime}} Y \subset \partial Y$ is the components of $\partial Y$ that $\alpha^{\prime}$ meets in its endpoints, and $\mathcal{N}($.$) denotes a regular neighborhood of$ their union (see [MM2, Sec. 2]).

When $A$ and $B$ are two subsets of $\mathcal{C}(Y)$, [MM2] defines a coarse distance $d_{Y}(A, B)$ by taking the diameter

$$
d_{Y}(A, B)=\operatorname{diam}_{\mathcal{C}(Y)}(A \cup B)
$$

in $\mathcal{C}(Y)$ of $A$ and $B$. Note that while $d_{Y}(.,$.$) is more a diameter than a distance$ when $A$ and $B$ are close, it gives a useful notion of distance between sets of bounded diameter and does satisfy the triangle inequality.

By [MM2, Lem. 2.3] the projection $\pi_{Y}$ has a Lipschitz property: if $\Delta$ is a simplex in $\mathcal{C}(S)$ so that $\Delta$ intersects $Y$, then we have $\operatorname{diam}_{\mathcal{C}(Y)}\left(\pi_{Y}(\Delta)\right) \leq 2$. If $P_{I}$ and $P_{T}$ are two subsets of $\mathcal{C}(S)$, letting $\pi_{Y}\left(P_{I}\right)=\bigcup_{\alpha \in P_{I}} \pi_{Y}(\alpha)$, and likewise for $P_{T}$, then the projection distance $d_{Y}\left(P_{I}, P_{T}\right)$ between $P_{I}$ and $P_{T}$ (or distance in $Y$ ) is defined by

$$
d_{Y}\left(P_{I}, P_{T}\right)=d_{Y}\left(\pi_{Y}\left(P_{I}\right), \pi_{Y}\left(P_{T}\right)\right) .
$$

In particular, if $P$ and $P^{\prime}$ are pants decompositions that differ by a single elementary move, then we have $d_{Y}\left(P, P^{\prime}\right) \leq 4$ (see [MM2 Lem. 2.5]).

A central theorem we will use is the following:

Theorem 4.4 (Thm. 6.12 of [MM2]). There is a constant $M_{0}(S)$ so that given $M>M_{0}$ there exist $c_{0}$ and $c_{1}$ so that if $P_{I}$ and $P_{T}$ are pants decompositions in $\mathbf{P}(S)$, then we have

$$
\frac{1}{c_{0}} d_{\mathbf{P}}\left(P_{I}, P_{T}\right)-c_{1} \leq \sum_{\substack{Y \subseteq S \\ d_{Y}\left(P_{I}, P_{T}\right)>M}} d_{Y}\left(P_{I}, P_{T}\right) \leq c_{0} d_{\mathbf{P}}\left(P_{I}, P_{T}\right)+c_{1},
$$

where the sum is taken over all non-annular essential subsurfaces $Y \subseteq S$ satisfying $d_{Y}\left(P_{I}, P_{T}\right)>M$.

We apply this result to prove Lemma 4.3. Our argument is quite similar to that of [MM2, Thm. 6.10], where it is shown that a given pants decomposition along an elementary move sequence can contribute to progress in only boundedly many projections to subsurfaces simultaneously. We seek the analogous statement for a single curve occurring in pants decompositions joining $P_{I}$ to $P_{T}$.

Proof of Lemma 4.3. To prove the lemma, we will relate the sum of the projections to the size of $\mathcal{S}_{g}$. To do this, we note that when the projection distance $d_{Y}\left(P_{I}, P_{T}\right)$ is large, there must be a definite portion of the projection of $g$ to $Y$ that is far from both $\pi_{Y}\left(P_{I}\right)$ and $\pi_{Y}\left(P_{T}\right)$ in $d_{Y}(.,$.$) ; this follows from the triangle inequality for d_{Y}$ and the fact that elementary moves in $\mathbf{P}(S)$ make Lipschitz progress as measured by $d_{Y}$. 
We argue that a given curve $\alpha$ can contribute only to a bounded amount of progress in boundedly many different subsurfaces. Precisely, let $Y$ and $Z$ be two essential, intersecting, non-annular subsurfaces of $S$, neither of which is contained in the other. A lemma of Masur and Minsky [MM2, Lem. 6.11] enforces a partial ordering " $\prec$ " on such subsurfaces with respect to the pants decompositions $P$ and $P^{\prime}$, provided the projection distances $d_{Y}\left(P, P^{\prime}\right)$ and $d_{Z}\left(P, P^{\prime}\right)$ are greater than a constant $M_{2}$ depending only on $S$. Taking $M_{2}$ to be the constant of [MM2, Lem. $6.2]$ with the same name, we say the subsurfaces $Y$ and $Z$ are $\left(P, P^{\prime}\right)$-ordered if we have

$$
d_{Y}\left(P, P^{\prime}\right)>M_{2} \text { and } d_{Z}\left(P, P^{\prime}\right)>M_{2} .
$$

We rephrase [MM2, Lem. 6.11] as follows.

Lemma 4.5. There is a constant $M_{3}$ depending only on $S$ so that if $Y$ and $Z$ are $\left(P, P^{\prime}\right)$-ordered, then one of two cases obtains. Either $Y \prec Z$, and we have

$$
d_{Y}\left(\partial Z, P^{\prime}\right)<M_{3} \text { and } d_{Z}(P, \partial Y)<M_{3}
$$

or $Z \prec Y$, and we have

$$
d_{Z}\left(\partial Y, P^{\prime}\right)<M_{3} \text { and } d_{Y}(P, \partial Z)<M_{3}
$$

Proof. If $Y$ and $Z$ are $\left(P, P^{\prime}\right)$-ordered in the above sense, then by [MM2, Lem. 6.2] they appear as domains $Y=D(h)$ and $Z=D(k)$ supporting tight geodesics $h \subset \mathcal{C}(Y)$ and $k \subset \mathcal{C}(Z)$ in any hierarchy $H$ (without annuli [MM2, Sec. 8]) joining $P=I(H)$ to $P^{\prime}=T(H)$. The condition that $Y$ and $Z$ intersect and are non-nested guarantees that $h$ and $k$ are time-ordered [MM2, Lem. 4.18] (in the sense of [MM2. Defn. 4.16]). The lemma then follows from an application of [MM2, Lem. 6.11], where $Y \prec Z$ represents the case $h \prec_{t} k$ and $Z \prec Y$ represents the case $k \prec_{t} h$.

Continuation of the proof of Lemma 4.3 Let $M_{4}=M_{2}+2 M_{3}+4$, and let $M=$ $\max \left\{4 M_{4}, M_{0}\right\}$. Consider an essential subsurface $Y \subseteq S$ for which $d_{Y}\left(P_{I}, P_{T}\right)>$ $M$. As in the proof of [MM2, Lem. 6.10] let $J_{Y}$ denote the subset of $[1, N]$ for which if $i \in J_{Y}$, then $P_{i}$ is "deep" in the projection to $Y$ : i.e.

$$
d_{Y}\left(P_{I}, P_{i}\right)>M_{4} \text { and } d_{Y}\left(P_{i}, P_{T}\right)>M_{4} .
$$

Given a subset $A \subset[1, N]$, we denote by

$$
\|A\|_{Y}=\operatorname{diam}_{\mathcal{C}(Y)}\left(\left\{\pi_{Y}\left(P_{i}\right) \mid i \in A\right\}\right)
$$

the diameter of the projection of the pants decompositions with indices in $A$ to the curve complex $\mathcal{C}(Y)$.

Given $\alpha \in \mathcal{S}_{g}$ for which $\pi_{Y}(\alpha) \neq \varnothing$, we denote by $J_{Y}(\alpha) \subset J_{Y}$ the subset for which if $i \in J_{Y}(\alpha)$, then $\alpha$ lies in $P_{i}$. We make three observations for later reference:

I. By the Lipschitz property for $\pi_{Y}$, we have $\left\|J_{Y}(\alpha)\right\|_{Y} \leq 4$.

II. If $i$ lies in $J_{Y}$ then there is some $\alpha \in \mathcal{S}_{g}$ so that $i \in J_{Y}(\alpha)$.

III. Since $d_{\mathbf{P}}\left(P_{j}, P_{j+1}\right)<k$, we have $d_{Y}\left(P_{j}, P_{j+1}\right)<4 k$.

Let $Y \subseteq S$ and $Z \subseteq S$ be two non-annular intersecting subsurfaces neither of which is contained in the other so that each contributes to the sum of Theorem 4.4 i.e. we have

$$
d_{Y}\left(P_{I}, P_{T}\right)>M \quad \text { and } \quad d_{Z}\left(P_{I}, P_{T}\right)>M
$$

This assumption guarantees, in particular, that $Y$ and $Z$ are $\left(P_{I}, P_{T}\right)$-ordered. We make the following claim: 
(*) If $J_{Y}(\alpha)$ is non-empty, then $J_{Z}(\alpha)$ must be empty.

Arguing by contradiction, assume

$$
J_{Y}(\alpha) \neq \varnothing \neq J_{Z}(\alpha) .
$$

We have

$$
\pi_{Y}(\alpha) \neq \varnothing \neq \pi_{Z}(\alpha)
$$

so if $i \in J_{Y}(\alpha)$ and $j \in J_{Z}(\alpha)$, then $\operatorname{diam}_{Y}\left(\pi_{Y}\left(P_{i}\right)\right) \leq 2$ and $\operatorname{diam}_{Y}\left(\pi_{Y}\left(P_{j}\right)\right) \leq 2$, so it follows that $d_{Y}\left(P_{i}, P_{j}\right) \leq 4$, since $\alpha$ lies in $P_{i}$ and in $P_{j}$. The same conclusion holds with $Z$ in place of $Y$.

Since $i$ lies in $J_{Y}(\alpha)$, we have $d_{Y}\left(P_{I}, P_{i}\right)>M_{4}$ and $d_{Y}\left(P_{i}, P_{T}\right)>M_{4}$, so it follows that

$$
d_{Y}\left(P_{I}, P_{j}\right) \geq M_{4}-4 \quad \text { and } \quad d_{Y}\left(P_{j}, P_{T}\right) \geq M_{4}-4 .
$$

As $j$ lies in $J_{Z}(\alpha)$ we have

$$
d_{Z}\left(P_{I}, P_{j}\right) \geq M_{4},
$$

and since $M_{4}-4>M_{2}$, it follows that $Y$ and $Z$ are also $\left(P_{I}, P_{j}\right)$-ordered. Let $\prec_{j}$ denote the $\left(P_{I}, P_{j}\right)$-ordering and assume without loss of generality that $Y \prec_{j} Z$. Then applying Lemma 4.5 we have

$$
d_{Y}\left(\partial Z, P_{j}\right)<M_{3} .
$$

Since $Y$ and $Z$ are also $\left(P_{I}, P_{T}\right)$-ordered, we may first assume that $Y \prec Z$. Then Lemma 4.5 gives $d_{Y}\left(\partial Z, P_{T}\right)<M_{3}$, which implies that

$$
d_{Y}\left(P_{j}, P_{T}\right)<2 M_{3},
$$

contradicting the assumption that $j \in J_{Z}(\alpha)$. If on the other hand we have $Z \prec Y$, then Lemma 4.5 gives $d_{Y}\left(P_{I}, \partial Z\right)<M_{3}$, from which we conclude

$$
d_{Y}\left(P_{I}, P_{j}\right)<2 M_{3}
$$

which contradicts the same assumption. Thus, either $J_{Y}(\alpha)$ or $J_{Z}(\alpha)$ must be empty, and the claim $(*)$ is proven.

Applying observations (I) and (II) above, if $\mathcal{S}_{Y}=\left\{\alpha \in \mathcal{S}_{g} \mid J_{Y}(\alpha) \neq \varnothing\right\}$, then we have

$$
\left\|J_{Y}\right\|_{Y} \leq 4\left|\mathcal{S}_{Y}\right|
$$

There is a uniform bound $s$ depending only on $S$ to the size of any collection of subsurfaces any pair of which is disjoint or nested (see [MM2, Lem. 6.10, proof]), so by our claim $(*)$, the number of $Y$ for which $J_{Y}(\alpha)$ can be non-empty is bounded by $s$. Thus we have

$$
\sum_{Y \subseteq S, J_{Y} \neq \varnothing}\left\|J_{Y}\right\|_{Y} \leq 4 s\left|\mathcal{S}_{g}\right| .
$$

Applying observation (III), we have $d_{Y}\left(P_{j}, P_{j+1}\right)<4 k$. Thus, for each $Y$ satisfying

$$
d_{Y}\left(P_{I}, P_{T}\right)>M
$$

the set $J_{Y}$ is in particular non-empty, and we have

$$
d_{Y}\left(P_{I}, P_{T}\right)-2 M_{4} \leq 4 k\left\|J_{Y}\right\|_{Y} .
$$

But since $4 M_{4}<M$, we have $2 M_{4} \leq 4 k\left\|J_{Y}\right\|_{Y}$ and thus

$$
d_{Y}\left(P_{I}, P_{T}\right) \leq 8 k\left\|J_{Y}\right\|_{Y} .
$$


Since $M=\max \left\{M_{0}, 4 M_{4}\right\}$, applying Theorem 4.4 there are constants $c_{0}$ and $c_{1}$ so that we have

$$
\frac{1}{c_{0}} d_{\mathbf{P}}\left(P_{I}, P_{T}\right)-c_{1} \leq \sum_{\substack{Y \subseteq S \\ d_{Y}\left(P_{I}, P_{T}\right)>M}} d_{Y}\left(P_{I}, P_{T}\right) \leq 32 s k\left|\mathcal{S}_{g}\right| .
$$

Since $\left|\mathcal{S}_{g}\right|$ is always at least $d(S)$, we may combine all of the above constants into a single $K_{0}$ for which

$$
d_{\mathbf{P}}\left(P_{I}, P_{T}\right) \leq K_{0}\left|\mathcal{S}_{g}\right|
$$

To prove Lemma 4.2, we will apply Lemma 4.3 to a sequence of pants decompositions $\left\{P_{X}=P_{0}, \ldots, P_{N}=P_{Y}\right\}$ so that $\pi_{s}\left(P_{j}\right) \subset \mathcal{S}_{<L}$ for each $j$, and so that $d_{\mathbf{P}}\left(P_{j}, P_{j+1}\right)$ is bounded by an a priori constant. The existence of such a sequence is provided by an interpolation of 1-Lipschitz homotopy equivalences of hyperbolic surfaces into $Q(X, Y)$ that pass from one side of the convex core to the other. The existence of such an interpolation follows from work of R. Canary on simplicial hyperbolic surfaces, which we now describe.

Simplicial hyperbolic surfaces. Let $\operatorname{Sing}_{k}(S)$ denote the finite-area marked singular hyperbolic structures on $S$ : complete finite area hyperbolic surfaces $Z$ with at most $k$ cone singularities, each with cone-angle at least $2 \pi$, equipped with marking homeomorphisms $h$ : $\operatorname{int}(S) \rightarrow Z$ up to marking-preserving isometry. Roughly speaking, a simplicial hyperbolic surface is a path-isometric mapping from a singular hyperbolic surface to a hyperbolic 3-manifold that is totally geodesic in the complement of a "triangulation." We now make this notion precise.

Let $V=\left\{v_{1}, \ldots, v_{p}\right\}$ be a finite subset of $S$. Following Hatcher Hat], an essential $\operatorname{arc} \alpha$ in $(S, V)$ is an embedded arc meeting $\partial S \cup V$ only in its endpoints, which lie in $V$. A collection $\left\{\alpha_{0}, \ldots, \alpha_{k}\right\}$ of essential arcs in $(S, V)$ that are pairwise embedded and non-isotopic rel-endpoints is called a curve system. Let $\mathcal{A}(S, V)$ denote the simplicial complex whose $k$-simplices $\left[\alpha_{0}, \ldots, \alpha_{k}\right]$ are curve systems with curves $\left\{\alpha_{0}, \ldots, \alpha_{k}\right\}$ with faces given by $k$-tuples of curves in $\left\{\alpha_{0}, \ldots, \alpha_{k}\right\}$.

If $V$ contains a point in each boundary component of the compact surface $S$, then a triangulation of $S$ is a maximal curve system in $\mathcal{A}(S, V)$. Likewise, we may view the interior $\operatorname{int}(S)$ of $S$ as a "punctured surface" by collapsing each boundary component $\gamma \subset \partial S$ to a point $v_{\gamma}$ to obtain a surface $R$. Then we have

$$
\operatorname{int}(S)=R-\left\{v_{\gamma} \mid \gamma \subset \partial S\right\}
$$

If $V$ is a subset of $R$ containing $\bigcup_{\gamma} v_{\gamma}$, then a triangulation of $\operatorname{int}(S)$ is the restriction of a maximal curve system in $\mathcal{A}(R, V)$ restricted to $R-\left\{v_{\gamma} \mid \gamma \subset \partial S\right\}=\operatorname{int}(S)$. Note that in each definition, an edge may have its boundary vertices identified and a face may have boundary edges identified.

The main result of [Hat] guarantees that any two triangulations in $\mathcal{A}(S, V)$ are related by a finite sequence of elementary moves (see Figure 5).

Let $T$ be a triangulation of $\operatorname{int}(S)$ in the above sense, and let $h: \operatorname{int}(S) \rightarrow Z$ be a singular hyperbolic surface for which $h$ is isotopic to a map with the property that each cone singularity of $Z$ lies in the image of a vertex of $T$. Isotope $h$ to send each edge of $T$ to its geodesic representative rel-endpoints on $Z$ (if an edge $e$ terminates at a puncture, $h$ should send $e$ to a geodesic arc asymptotic to the corresponding cusp of $Z$ ). Then if $N$ is a hyperbolic 3-manifold and there is a 

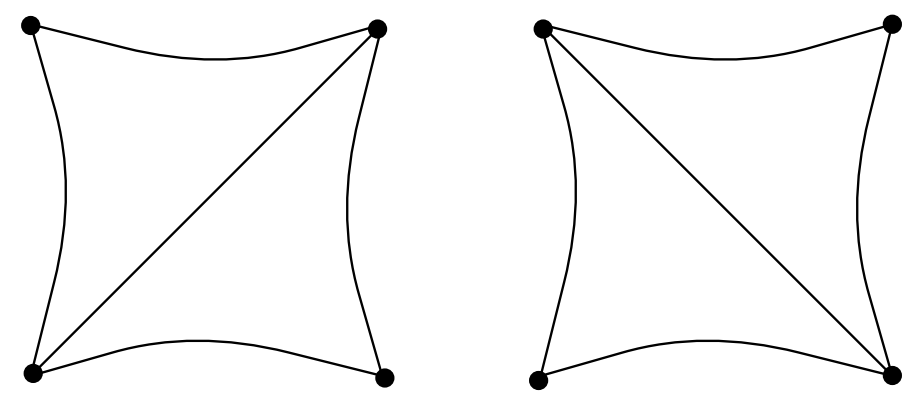

FiguRE 5. Elementary moves on triangulations.

path-isometry $g: Z \rightarrow N$ that is a local isometry on $Z-T$, then we call the pair $(g, Z)$ a simplicial hyperbolic surface in $N$ with associated triangulation $T$.

Often the construction goes in the other direction: given a triangulation $T$ of $\operatorname{int}(S)$ with one vertex $v \in \operatorname{int}(S)$ and at least one edge $e$ so that $e \cup v$ forms a closed loop $\gamma$, we can straighten any smooth, proper, incompressible map $g^{\prime}$ of $\operatorname{int}(S)$ to $N$ to a simplicial hyperbolic surface with associated triangulation $T$. First we straighten $g^{\prime}$ so it maps $\gamma$ to its geodesic representative. Then, straightening $g^{\prime}$ on the edges of $T$ rel-endpoints (possibly ideal endpoints) and then on faces of $T$, we obtain a map $g: \operatorname{int}(S) \rightarrow N$. The pull-back metric from $N$ determines a singular hyperbolic surface $Z$ with a cone singularity at $v$; since $v$ lies on a closed geodesic that is mapped to a closed geodesic in $N$, the cone angle at $v$ is at least $2 \pi$. The result is a simplicial hyperbolic surface $(g, Z)$ in $N$ with associated triangulation $T$. In this case we say that $(g, Z)$ is adapted to $\gamma$.

Let $(f: S \rightarrow N) \in A H(S)$. Then we denote by $\mathcal{S H}_{k}(N)$ the marking preserving simplicial hyperbolic surfaces in $N$ with at most $k$ cone-singularities, namely, simplicial hyperbolic surfaces $(g, Z)$, with $(h: \operatorname{int}(S) \rightarrow Z) \in \operatorname{Sing}_{k}(S)$, so that $g \circ h$ is homotopic to $f$. If $\sigma$ is a simplex in $\mathcal{C}(S)$ with vertices determined by curves $\alpha_{1}, \ldots, \alpha_{p}$, then we say a simplicial hyperbolic surface $(g, Z) \in \mathcal{S H}_{k}(N)$ realizes $\sigma$ if $g$ maps each $\alpha_{i}$ isometrically to its geodesic representative in $N$.

Recall that a manifold $(f: S \rightarrow N) \in A H(S)$ has an accidental parabolic if there is a non-peripheral element $\gamma \in \pi_{1}(S)$ so that $f_{*}(\gamma)$ is a parabolic element of $\operatorname{Isom}^{+}\left(\mathbb{H}^{3}\right)$. Applying Hatcher's theorem [Hat] allowing one to connect triangulations by elementary moves, Canary proves the following (see [Can3, §5, §6]; compare [Br1, §3] and [Min, §4]):

Theorem 4.6 (Canary). Let $N \in A H(S)$ have no accidental parabolics, and let $\left(g_{1}, Z_{1}\right)$ and $\left(g_{2}, Z_{2}\right)$ lie in $\mathcal{S} \mathcal{H}_{1}(N)$ where $\left(g_{1}, Z_{1}\right)$ is adapted to $\alpha$ and $\left(g_{2}, Z_{2}\right)$ is adapted to $\beta$. Then there is a continuous family $\left(g_{t}: Z_{t} \rightarrow N\right) \subset \mathcal{S H}_{2}(N), t \in[1,2]$.

Using such an interpolation, we now give the proof of Lemma 4.2

Proof of Lemma 4.2. Given the quasi-Fuchsian manifold $Q(X, Y)$, let $P_{X}$ and $P_{Y}$ denote pants decompositions for which $X$ lies in $V\left(P_{X}\right)$ and $Y$ lies in $V\left(P_{Y}\right)$. Then by a theorem of Bers ([Brs2, Thm. 3], Mc1 Prop. 6.4]), we have

$$
\ell_{Q(X, Y)}(\alpha)<2 L
$$

for each curve $\alpha$ in $P_{X} \cup P_{Y}$. 
Let $Z_{X}$ and $Z_{Y}$ denote simplicial hyperbolic surfaces realizing $P_{X}$ and $P_{Y}$ in $Q(X, Y)$ and let $T_{X}$ and $T_{Y}$ denote their associated triangulations. Let $Z_{X}^{\mathrm{h}} \in$ Teich $(S)$ be the hyperbolic surface conformally equivalent to $Z_{X}$, and let $Z_{Y}^{\mathrm{h}}$ be the hyperbolic surface conformally equivalent to $Z_{Y}$. Finally, let $P_{I}$ and $P_{T}$ be pants decompositions so that $Z_{X}^{\mathrm{h}} \in V\left(P_{I}\right)$ and $Z_{Y}^{\mathrm{h}} \in V\left(P_{T}\right)$.

The next step will be to interpolate simplicial hyperbolic surfaces between $Z_{X}$ and $Z_{Y}$ and estimate the minimum number of sets $V\left(P_{j}\right)$ the corresponding conformally equivalent hyperbolic representatives in $\operatorname{Teich}(S)$ intersect. To show we have not sacrificed too much distance in the pants graph, we prove the following:

Lemma 4.7. There is a constant $B^{\prime}>0$ depending only on $S$ so that

$$
\max \left\{d_{\mathbf{P}}\left(P_{X}, P_{I}\right), d_{\mathbf{P}}\left(P_{Y}, P_{T}\right)\right\}<B^{\prime} .
$$

Proof. We have

$$
\ell_{Z_{X}}(\gamma)<2 L
$$

for each $\gamma \in P_{X}$. By a lemma of Ahlfors [Ah] we have

$$
\ell_{Z_{X}^{\mathrm{h}}}(\beta) \geq \ell_{Z_{X}}(\beta)
$$

for each $\beta \in \mathcal{S}$. Thus, we have

$$
\ell_{Z_{X}}(\alpha)<2 L
$$

for each $\alpha \in P_{X} \cup P_{I}$. Since curves in $P_{X} \cup P_{I}$ are realized with bounded length on the simplicial hyperbolic surface $Z_{X} \in \mathcal{S H}_{k}(Q(X, Y))$, we may apply Br1, Lem. 3.3 to find a $C^{\prime}>0$ depending only on $L$ so that

$$
i\left(P_{X}, P_{I}\right)<C^{\prime}
$$

Arguing similarly for $Z_{Y}$, we have

$$
i\left(P_{Y}, P_{T}\right)<C^{\prime} .
$$

Applying the proof of Theorem 3.3 we have a $B^{\prime}>0$ depending only on $C^{\prime}$ for which

$$
\max \left\{d_{\mathbf{P}}\left(P_{X}, P_{I}\right), d_{\mathbf{P}}\left(P_{Y}, P_{T}\right)\right\}<B^{\prime} .
$$

Continuation of the proof of Lemma 4.2. To complete the proof of Lemma 4.2 we seek a continuous family

$$
\left(h_{t}: Z_{t} \rightarrow Q(X, Y)\right) \subset \mathcal{S H}_{k}(Q(X, Y))
$$

of simplicial hyperbolic surfaces in $Q(X, Y)$ interpolating between $\left(h_{X}, Z_{X}\right)$ and $\left(h_{Y}, Z_{Y}\right)$. We first connect $\left(h_{X}, Z_{X}\right)$ and $\left(h_{Y}, Z_{Y}\right)$ to simplicial hyperbolic surfaces $\left(h_{X}^{\prime}, Z_{X}^{\prime}\right)$ and $\left(h_{Y}^{\prime}, Z_{Y}^{\prime}\right)$ adapted to single curves $\alpha \in P_{X}$ and $\beta \in P_{Y}$ by continuous families, and then apply the interpolation arguments of Canary.

This is easily done by collapsing edges of $T_{X}$ that join distinct vertices of $T_{X}$ down to a single vertex: if $e$ is such an edge adjacent to a vertex $v$ on $\alpha$, then we may effect such a collapsing by 'dragging $h_{X}$ along $h_{X}(e)$ ' (see [Can3, §5]). Precisely, if $v^{\prime}$ is the other vertex in $\partial e$, we construct a homotopy of $\left(h_{X}, Z_{X}\right)$ to a new simplicial hyperbolic surface by pulling the image $h_{X}\left(v^{\prime}\right)$ along the geodesic segment $h_{X}(e)$ to $h_{X}(v)$ and pulling the edges and faces adjacent to $v$ along with it while keeping the triangulation straight: the image of each triangle is required to lift to the convex hull of its vertices throughout the homotopy. 
It is easy to check that under such a collapsing the cone angles at the vertices remain at least $2 \pi$, and the number of vertices in $T$ is reduced by 1 . We successively collapse edges joining $v$ to different vertices until we are left with a triangulation with the single vertex $v$, and a simplicial hyperbolic surface $\left(h_{X}^{\prime}, Z_{X}^{\prime}\right) \in$ $\mathcal{S H}_{1}(Q(X, Y))$ adapted to $\alpha$.

We perform analogous collapsings on the associated triangulation for $\left(h_{Y}, Z_{Y}\right)$ to obtain the simplicial hyperbolic surface $\left(h_{Y}^{\prime}, Z_{Y}^{\prime}\right) \in \mathcal{S H}_{1}(Q(X, Y))$ adapted to $\beta$. By Theorem 4.6, we may interpolate between $\left(h_{X}^{\prime}, Z_{X}^{\prime}\right)$ and $\left(h_{Y}^{\prime}, Z_{Y}^{\prime}\right)$ by a continuous family of simplicial hyperbolic surfaces, so we have the desired continuous family

$$
\left(h_{t}: Z_{t} \rightarrow C(X, Y)\right) \subset \mathcal{S H}_{k}(Q(X, Y)), \quad t \in[0,1]
$$

so that $\left(h_{0}, Z_{0}\right)=\left(h_{X}, Z_{X}\right)$ and $\left(h_{1}, Z_{1}\right)=\left(h_{Y}, Z_{Y}\right)$.

The singular hyperbolic structures $Z_{t}$ determine a continuous path

$$
\left(h_{t}^{\mathrm{h}}: \operatorname{int}(S) \rightarrow Z_{t}^{\mathrm{h}}\right) \subset \operatorname{Teich}(S),
$$

where as before $Z_{t}^{\mathrm{h}}$ is the finite-area hyperbolic structure on $S$ in the same conformal class as $Z_{t}$. Since $Z_{t}$ and $Z_{t}^{\mathrm{h}}$ represent metrics on the same underlying surface $\operatorname{int}(S)$, we have a natural continuous family of 1-Lipschitz mappings

$$
\hat{h_{t}}: Z_{t}^{\mathrm{h}} \rightarrow C(X, Y)
$$

of hyperbolic surfaces $Z_{t}^{\mathrm{h}} \in \operatorname{Teich}(S)$ into $Q(X, Y)$ so that for each $t, \hat{h}_{t}$ factors through the simplicial hyperbolic surface $h_{t}: Z_{t} \rightarrow C(X, Y)$.

There are pants decompositions $P_{1}, \ldots, P_{N}$ (possibly with repetition) that determine an open cover $\left\{U_{j}\right\}_{j=0}^{N}$ of $[0,1]$ so that if $t \in U_{j}$ then $Z_{t}^{\mathrm{h}}$ lies in $V\left(P_{j}\right)$, and so that $U_{j} \cap U_{j+1} \neq \varnothing$ for each $j=0, \ldots, N-1$. Applying Lemma [3.3 the sequence of pants decompositions

$$
g=\left\{P_{I}=P_{0}, \ldots, P_{N}=P_{T}\right\}
$$

satisfies the hypotheses of Lemma 4.3 with $k=B$. Applying Lemma 4.3, we have a $K_{0}$ so that

$$
d_{\mathbf{P}}\left(P_{I}, P_{T}\right) \leq K_{0}\left|\mathcal{S}_{g}\right|
$$

Since the non-empty set $\mathcal{S}_{g}$ is a subset of $\mathcal{S}_{<L}$, and Lemma 4 .7 guarantees

$$
d_{\mathbf{P}}\left(P_{I}, P_{T}\right) \geq d_{\mathbf{P}}\left(P_{X}, P_{Y}\right)-2 B^{\prime}
$$

we may combine constants to obtain a $K$ for which

$$
d_{\mathbf{P}}\left(P_{X}, P_{Y}\right) \leq K\left|\mathcal{S}_{<L}\right|
$$

proving the lemma.

Given a hyperbolic 3 -manifold $M$, we let $\mathcal{G}_{<L}(M)$ denote the set of homotopy classes of closed geodesics in $M$ with length bounded above by a constant $L>0$. We use the contraction $\mathcal{G}_{<L}=\mathcal{G}_{<L}(M)$ when the manifold $M$ is understood. The next lemma shows that the size $\left|\mathcal{G}_{<L}\right|$ of $\mathcal{G}_{<L}$ provides a lower bound for the convex core volume of a hyperbolic 3-manifold in a general context.

Lemma 4.8. Let $M$ be a geometrically finite hyperbolic 3-manifold with $\partial M$ incompressible, and let $\operatorname{vol}(M)$ denote its convex core volume. Then there is a constant $C_{1}>1$ depending only on $L$ and $C_{2}>0$ depending only on $\chi(\partial M)$ for which

$$
\frac{\left|\mathcal{G}_{<L}\right|}{C_{1}}-C_{2}<\operatorname{vol}(M)
$$


Proof. Let $\epsilon>0$ be less than the minimum of $L / 2$ and the 3-dimensional Margulis constant. Let $\mathcal{V}$ be any maximal set of points in the $\epsilon$-thick part $\operatorname{core}(M)_{\geq \epsilon}$ of the convex core $\operatorname{core}(M)$ for which points in $\mathcal{V}$ are separated by a distance at least $\epsilon / 2$. Letting $B(x, R)$ denote the ball of radius $R$ about $x$ in $M$, it follows that $B(x, \epsilon / 4)$ is embedded in the $\epsilon$-neighborhood $\mathcal{N}_{\epsilon}\left(\operatorname{core}(M)_{\geq \epsilon}\right)$ of the $\epsilon$-thick part of $M$, and

$$
B(x, \epsilon / 4) \cap B\left(x^{\prime}, \epsilon / 4\right)=\varnothing
$$

for $x \neq x^{\prime}$ in $\mathcal{V}$. By maximality, however, we have

$$
\operatorname{core}(M)_{\geq \epsilon} \subset \bigcup_{x \in \mathcal{V}} B(x, \epsilon / 2) .
$$

Each isotopy class $\beta \in \mathcal{G}_{<L}$ has a representative $\beta^{\star} \subset \operatorname{core}(M)_{\geq \epsilon}$ with arclength less than $L$. Since the $\epsilon / 2$-balls about points in $\mathcal{V}$ cover core $(M)_{\geq \epsilon}$, each $\beta^{\star}$ intersects $B(x, \epsilon / 2)$ for some $x \in \mathcal{V}$. Given $x \in \mathcal{V}$, let $\mathcal{A}_{x} \subset \mathcal{G}_{<L}$ denote the set

$$
\mathcal{A}_{x}=\left\{\beta \in \mathcal{G}_{<L} \mid \beta^{\star} \cap B(x, \epsilon / 2) \neq \varnothing\right\} .
$$

Lifting to the universal cover so that $x$ lifts to the origin $0 \in \mathbb{H}^{3}$, the elements $\beta \in \mathcal{A}_{x}$ determine pairwise disjoint translates of the ball $B(0, \epsilon / 2) \subset \mathbb{H}^{3}$ lying within the ball $B(0, L+2 \epsilon) \in \mathbb{H}^{3}$. It follows that the number of elements in each $\mathcal{A}_{x}$ satisfies

$$
\left|\mathcal{A}_{x}\right|<\frac{\operatorname{vol}(B(0, L+2 \epsilon))}{\operatorname{vol}(B(0, \epsilon / 2))},
$$

which we set equal to $C_{0}$.

Since every $\beta \in \mathcal{G}_{<L}$ lies in some $\mathcal{A}_{x}$, we have

$$
\frac{\left|\mathcal{G}_{<L}\right|}{C_{0}} \leq|\mathcal{V}| .
$$

Since the balls of radius $\epsilon / 4$ about points $x \in \mathcal{V}$ are embedded and pairwise disjoint in $\mathcal{N}_{\epsilon}\left(\operatorname{core}(M)_{\geq \epsilon}\right)$, we have the lower bound

$$
\frac{\left|\mathcal{S}_{<L}\right|}{C_{0}} \cdot \operatorname{vol}(B(0, \epsilon / 4)) \leq \operatorname{vol}_{\epsilon}(M),
$$

where $\operatorname{vol}_{\epsilon}(M)=\operatorname{vol}\left(\mathcal{N}_{\epsilon}(\operatorname{core}(M))\right)$. There is a constant $K_{\epsilon}>0$ depending only on $\epsilon$ and $M$ so that

$$
\operatorname{vol}_{\epsilon}(M)-\operatorname{vol}(M)<K_{\epsilon}
$$

(see e.g. Can2, Lem. 8.2], [Th1, 8.12.1]). The lemma follows by setting $C_{1}=$ $C_{0} / \operatorname{vol}(B(0, \epsilon / 4))$ and $C_{2}=K_{\epsilon}$.

Proof of Theorem 4.1. Since $\mathcal{S}_{<L}$ is a subset of $\mathcal{G}_{<L}(Q(X, Y))$, we may combine Lemma 4.2 with Lemma 4.8 to obtain

$$
\frac{d_{\mathbf{P}}\left(P_{X}, P_{Y}\right)}{K \cdot C_{1}}-C_{2} \leq \operatorname{vol}(X, Y) .
$$

Applying Theorem 3.2 we have

$$
\frac{d_{\mathrm{WP}}(X, Y)}{A_{1} \cdot K \cdot C_{1}}-\frac{A_{2}}{K \cdot C_{1}}-C_{2} \leq \operatorname{vol}(X, Y) .
$$

Letting

$$
K_{1}=A_{1} \cdot K \cdot C_{1} \quad \text { and } \quad K_{2}=\frac{A_{2}}{K \cdot C_{1}}+C_{2}
$$

the theorem follows. 


\section{Bounding the CORE VOlume From ABOve}

Our goal in this section will be to prove the following theorem.

Theorem 5.1. Given $S$, there are constants $K_{3}$ and $K_{4}$ so that if $Q(X, Y) \in$ $Q F(S)$ is a quasi-Fuchsian manifold and $P_{X}$ and $P_{Y}$ are pants decompositions for which $X \in V\left(P_{X}\right)$ and $Y \in V\left(P_{Y}\right)$, then we have

$$
\operatorname{vol}(X, Y) \leq K_{3} d_{\mathbf{P}}\left(P_{X}, P_{Y}\right)+K_{4} .
$$

Given Theorem 4.1 and Theorem [3.2, Theorem 5.1 represents the final step in the proof of Theorem 1.2

Let $G \subset \mathbf{P}(S)$ be a shortest path joining $P_{X}$ and $P_{Y}$ so that the length of $G$ is simply $d_{\mathbf{P}}\left(P_{X}, P_{Y}\right)$. Let

$$
\operatorname{spin}(G)=\left\{\alpha^{*} \mid \alpha \in P, P \in G\right\}
$$

denote the geodesic representatives in $Q(X, Y)$ of elements of the pants decompositions along $G$. We call these geodesics the spinning geodesics for $G$; they will serve to anchor various tetrahedra in $Q(X, Y)$ at their vertices; we will then "spin" these tetrahedra by pulling their vertices around the geodesics.

Our upper bound for $\operatorname{vol}(X, Y)$ will come from a model manifold $N=S \times I$ comprised of blocks that are adapted to $\operatorname{spin}(G)$, together with a piecewise $C^{1}$ surjective homotopy equivalence $f: N \rightarrow \mathcal{N}_{\epsilon}(C(X, Y))$ so that the image of each block under $f$ has uniformly bounded volume. The model will decompose into two parts.

(1) The Caps: At each end of $N$ are caps, namely products $S \times I$ on which $f$ restricts to homotopies of simplicial hyperbolic surfaces

$$
h_{X}: Z_{X} \rightarrow C(X, Y) \text { and } h_{Y}: Z_{Y} \rightarrow C(X, Y)
$$

realizing $P_{X}$ and $P_{Y}$ to the boundary components $X_{h}^{\epsilon}$ and $Y_{h}^{\epsilon}$ of the $\epsilon$ neighborhood $\mathcal{N}_{\epsilon}(C(X, Y))$ of the convex core.

(2) The Triangulated Part: The caps sit at either end of the triangulated part $N_{\Delta}$, a union of tetrahedra on which $f$ is simplicial: $f$ lifts to a map sending each simplex to the convex hull of its vertices. It follows that the image of each tetrahedron $\Delta \in N_{\Delta}$ under $f$ has uniformly bounded volume. We use the geodesics $\alpha^{*}$, where $\alpha \in P \in G$, as a scaffolding to build $N_{\Delta}$, a gluing of tetrahedra whose image interpolates between the simplicial hyperbolic surfaces $Z_{X}$ and $Z_{Y}$. After "spinning" $f$ sufficiently far about the spinning geodesics, all but a constant times $d_{\mathbf{P}}\left(P_{X}, P_{Y}\right)$ of the tetrahedra in $N_{\Delta}$ have images with small volume.

These two arguments give the desired bound after collecting constants.

Remark. The above spinning trick is inspired by the ideal simplicial maps of Th2, which are in effect a limit of the spinning process we perform here. The result in our context of passing to such a limit is an ideal triangulation of all but a bounded volume portion of $C(X, Y)$, with a uniformly bounded number of ideal tetrahedra necessary to accomplish each individual elementary move (the small volume tetrahedra collapse to lower-dimensional ideal edges and faces). We have chosen to work with finite triangulations in the interest of demonstrating how the 

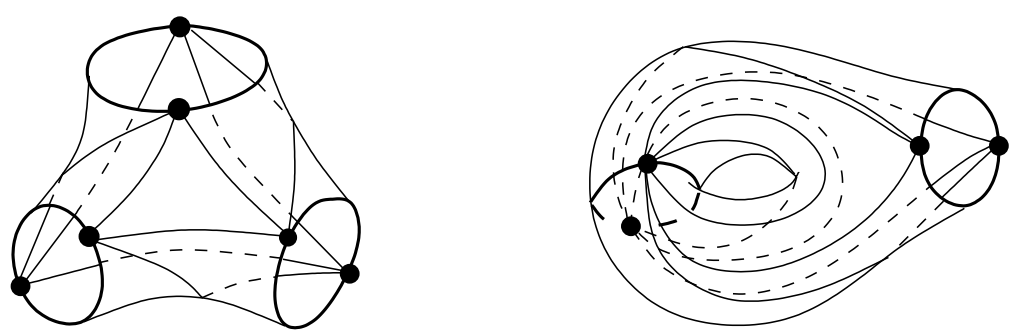

FiguRE 6. Standard triangulations suited to a pants decomposition.

combinatorics of $\mathbf{P}(S)$ may be used to produce triangulations of 3-manifolds in semi-algorithmic manner, independent of any geometric structure.

5.1. Triangulations of surfaces. We specify a type of triangulation of $S$ that is suited to a pants decomposition $P$. By a pair of pants we will mean a connected component $\widehat{S}$ of $S-\mathcal{N}(P)$, the complement of the union of pairwise disjoint open annular neighborhoods $\mathcal{N}(P)$ of the curves in $P$ on $S$.

Definition 5.2. A standard triangulation $T(\widehat{S})$ for a pair of pants $\widehat{S}$ is a triangulation with the following properties:

(1) $T(\widehat{S})$ has two vertices on each boundary component.

(2) $T(\widehat{S})$ has two disjoint spanning triangles with no vertices in common, and a vertex on each component of $\partial \widehat{S}$.

(3) The remaining 3 quadrilaterals are diagonally subdivided by an arc that travels "left to right" with respect to the inward pointing normal to $\partial \widehat{S}$ (see Figure 6).

We construct a standard triangulation suited to a pants decomposition $P \in \mathbf{P}(S)$ by gluing together standard triangulations on pairs of pants $\widehat{S}$ as follows.

Definition 5.3. Given a pants decomposition $P \in \mathbf{P}(S)$, a standard triangulation suited to $P$ is a triangulation $T$ of $S$ obtained as follows (see Figure 6):

(1) $T$ has two vertices $p_{\alpha}$ and $\bar{p}_{\alpha}$ on each component $\alpha$ of $P$, and two edges $e_{\alpha}$ and $\bar{e}_{\alpha}$ in the complement $\alpha-p_{\alpha} \cup \bar{p}_{\alpha}$.

(2) If $S_{0}$ is a complementary open pair of pants in $S-P$, then the restriction of $T$ to $S_{0}$ is the restriction to $S_{0}$ of a standard triangulation of $S_{0} \cup \partial S_{0}$ (in the sense of Definition 5.2 .

(3) If two boundary components $\alpha_{1}$ and $\alpha_{2}$ of $S_{0}$ are identified in $S$, then the edge of each spanning triangle that runs from $\alpha_{1}$ to $\alpha_{2}$ forms a closed loop.

Moves on triangulations. Given an elementary move on pants decompositions $\left(P, P^{\prime}\right)$, i.e. $d_{\mathbf{P}}\left(P, P^{\prime}\right)=1$, we now describe simple moves on triangulations that allow us to move from a standard triangulation suited to $P$ to a standard triangulation suited to $P^{\prime}$. To distinguish moves on triangulations from moves on pants decompositions, we refer to the latter as pants moves.

To fix notation, given a pants move $\left(P, P^{\prime}\right)$ let $\alpha \in P$ and $\beta \in P^{\prime}$ be the curves for which $i(\alpha, \beta) \neq 0$. We call $\alpha$ and $\beta$ the curves involved in the pants move $\left(P, P^{\prime}\right)$. Let $\mathcal{N}(P-\alpha)$ denote the union of pairwise disjoint open annular neighborhoods about the curves in $P-\alpha$, and let $S_{\alpha}$ denote the essential subsurface 
of $S-\mathcal{N}(P-\alpha)$ containing $\alpha$. If $S_{\alpha}$ has genus 1 , then $\left(P, P^{\prime}\right)$ is called a genus 1 pants move. Likewise, if $S_{\alpha}$ has genus 0 , then $\left(P, P^{\prime}\right)$ is called a genus 0 pants move. We say the pants move $\left(P, P^{\prime}\right)$ occurs on $S_{\alpha}$.

A standard triangulation $T$ suited to $P$ naturally identifies candidate elementary moves for each $\alpha \in P$ : there is a natural choice of isotopy class of simple closed curves $\beta \subset S_{\alpha}$ for which $i(\alpha, \beta)=1$ or $i(\alpha, \beta)=2$ depending on whether $S_{\alpha}$ has genus 1 or genus 0 . If $S_{\alpha}$ has genus 1, then each spanning triangle for $T$ in $S_{\alpha}$ has one edge with its endpoints identified. These edges are in the same isotopy class, which we call $\beta(\alpha, T)$. Likewise, if $S_{\alpha}$ has genus 0 , then removing the edges of $T$ that do not have endpoints lying on $\alpha$ produces two hexagons in the complement of the remaining edges. Concatenating edges in these hexagons joining the two vertices in each that lie on $\alpha$ we obtain an isotopy class of simple closed curves, which we again call $\beta(\alpha, T)$.

For each $\alpha \in P$, the pants decomposition $P^{\prime}=(P-\alpha) \cup \beta(\alpha, T)$ satisfies $d_{\mathbf{P}}\left(P, P^{\prime}\right)=1$.

There are three basic types of moves on these triangulations:

MVI. The Dehn twist move. One standard move on triangulations we will use effects a Dehn twist of a standard triangulation suited to $P$ about a curve $\alpha \in P$. Given a triangulation $T$ suited to $P$, let $T W_{\alpha}(T)$ denote the standard triangulation suited to $P$ obtained by shifting each edge with a vertex on $\alpha$ to the right along $\alpha$ until it hits the next vertex. Then $T W_{\alpha}(T)$ is isotopic to the image of $T$ under a right Dehn twist about $\alpha$. We define $T W_{\alpha}^{-1}(T)$ similarly, by shifting edges to the left rather than to the right.

Note that for any triangulation $T$ suited to $P$ and any $\alpha \in P$ we have

$$
\beta\left(\alpha, T W_{\alpha}(T)\right)=\tau_{\alpha}(\beta(\alpha, T)),
$$

where $\tau_{\alpha}$ is a right Dehn twist about $\alpha$.

The other elementary moves on triangulations will be specific to a given type of pants move. Given a triangulation $T$ suited to $P$ and $\alpha \in P$, we describe blowdown and blow-up moves that allow us to pass from a triangulation suited to $P$ to a triangulation suited to the pants decomposition $(P-\alpha) \cup \beta(\alpha, T)$.

MVII. Genus 1 moves. Given a standard triangulation $T$ suited to $P$ the edges of $T$ that close to form loops in the isotopy class of $\beta(\alpha, T)$ bound an annulus on $S$. We call this annulus $A_{\alpha}$ the $\alpha$ compressing annulus for $T$. The genus $1 \alpha$-blow-down $B D_{\alpha}^{1}(T)$ of $T$ is the triangulation of $S$ obtained by collapsing the arc $e$ of $\alpha \cap A_{\alpha}$ to a point and collapsing the two triangles in $T$ containing $e$ to a single edge.

Let $\beta=\beta(\alpha, T)$. Then given $T^{\prime}=B D_{\alpha}^{1}(T)$ the genus $1 \beta$-blow-up $B U_{\beta}^{1}\left(T^{\prime}\right)$ of $T^{\prime}$ is obtained by grafting a compressing annulus $A_{\beta}$ in along the curve $\alpha$ to obtain a standard triangulation suited to $(P-\alpha) \cup \beta$ as in Figure 7

MVIII. Genus 0 moves. Let $T$ be a standard triangulation suited to $P$. Let $e_{\alpha}$ and $\bar{e}_{\alpha}$ denote the two edges of $T$ that constitute the curve $\alpha$. Call these curves the $\alpha$-edges of $T$. As described above there are two hexagons $H$ and $\bar{H}$ obtained by removing all edges of $T$ that do not have endpoints on $\alpha$. The genus $0 \alpha$-blow-down $B D_{\alpha}^{0}(T)=T^{\prime}$ (Figure 9) is obtained by performing 3 "diagonal switches" on each hexagon (Figure 8) to yield a new triangulation with edges $e_{\beta}$ and $\bar{e}_{\beta}$ as edges whose concatenation gives the curve $\beta=\beta(\alpha, T)$. 

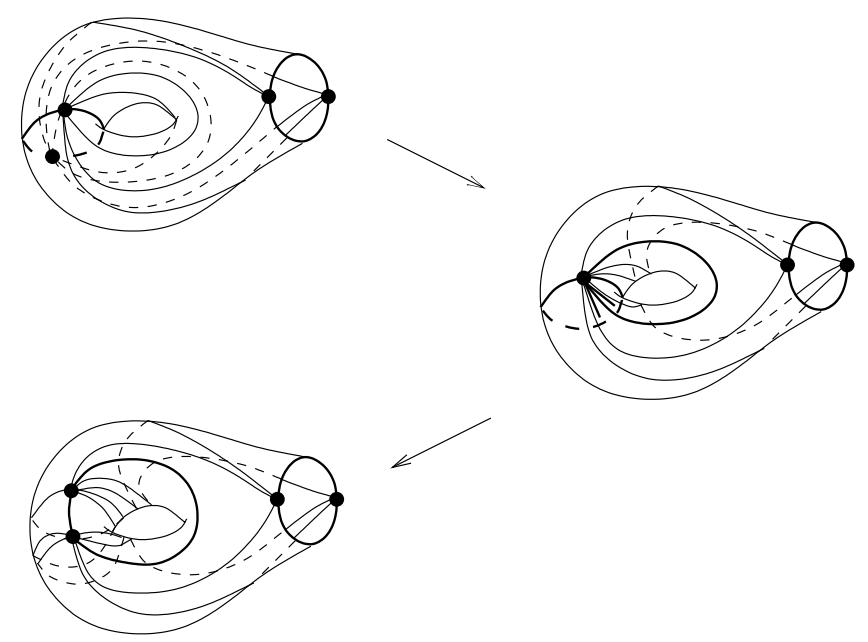

Figure 7 . The genus 1 blow-down and blow-up moves.
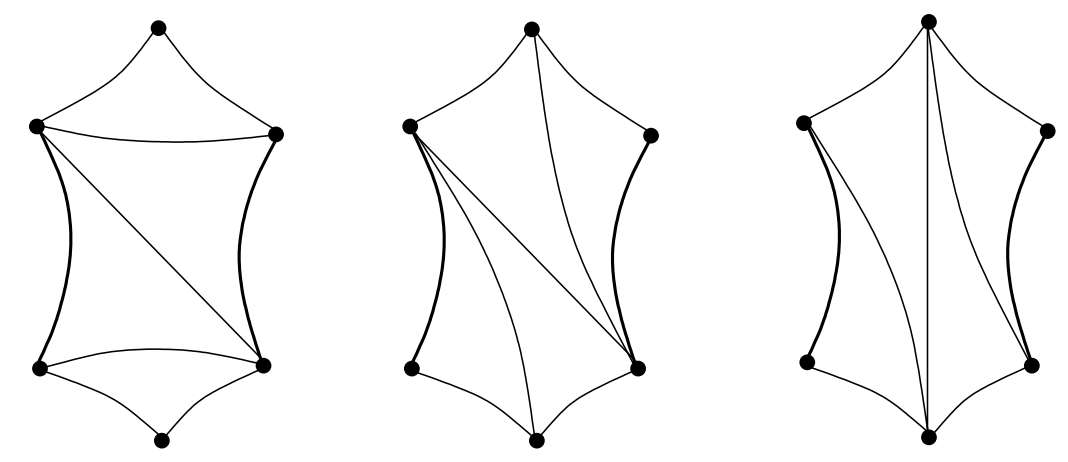

FiguRE 8. Diagonal switches on hexagons.
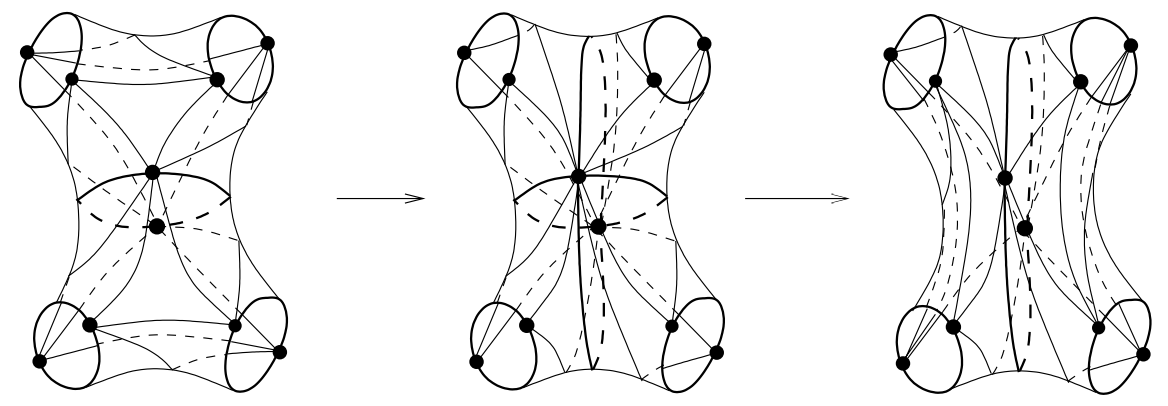

Figure 9. The genus 0 blow-down and blow-up moves.

Likewise, the genus $0 \beta$-blow-up $B U_{\beta}^{1}\left(T^{\prime}\right)$ is obtained by modifying the hexagons containing the $\alpha$-edges by the inverses of the 3 -diagonal switches. Note that $B U_{\beta}^{0}$ 。 $B D_{\alpha}^{0}(T)$ is a standard triangulation suited to the pants decomposition $(P-\alpha) \cup \beta$. 
We summarize properties of these moves as a lemma.

Lemma 5.4. Let $\left(P, P^{\prime}\right)$ be a pants move involving $\alpha \in P$ and $\alpha^{\prime} \in P^{\prime}$. Let $T$ be a standard triangulation suited to $P$. Then there is an $n \in \mathbb{Z}$ so that

$$
B U_{\alpha^{\prime}} \circ B D_{\alpha} \circ T W_{\alpha}^{n}(T)
$$

is a standard triangulation suited to $P^{\prime}$.

5.2. Realizing moves by blocks. As before let $Q(X, Y)$ be a quasi-Fuchsian manifold, and let $P_{X}$ and $P_{Y}$ be pants decompositions for which $X \in V\left(P_{X}\right)$ and $Y \in V\left(P_{Y}\right)$. Let $G \subset \mathbf{P}(S)$ be a geodesic joining $P_{X}$ and $P_{Y}$. Recall we denote by $\operatorname{spin}(G)=\left\{\alpha^{*} \mid \alpha \in P, P \in G\right\}$ the spinning geodesics associated to $G$.

Equip each spinning geodesic $\alpha^{*}$ with a pair of antipodal vertices $p_{\alpha}$ and $\bar{p}_{\alpha}$ : i.e. points on $\alpha^{*}$ so that the distance from $p_{\alpha}$ to $\bar{p}_{\alpha}$ along $\alpha^{*}$ is maximal. For reference, we equip each $\alpha$ and thence each $\alpha^{*}$ with an orientation.

Let $P_{i}, i=0, \ldots, m$, denote the pants decompositions along the geodesic $G$, so that $P_{0}=P_{X}$ and $P_{m}=P_{Y}$. Making an initial choice of standard triangulation $T_{X}$ suited to $P_{X}$, Lemma 5.4 provides a sequence of moves on triangulations allowing us to process from the triangulation $T_{X}$ to a standard triangulation $T_{Y}$ suited to $Y$ via standard triangulations $T_{i}$ suited to $P_{i}$. We begin with a model manifold

$$
N_{0} \cong S \times I
$$

and triangulate $\partial^{+} N_{0}=S \times\{1\}$ by $T_{X}$. Our aim is to build models $N_{i} \cong S \times I$ by gluing triangulated I-bundles to $\partial^{+} N_{i-1}$ so that the resulting triangulation on $\partial^{+} N_{i}$ is $T_{i}$. We do this by building a triangulated subsurface block corresponding to each elementary move and successively gluing the blocks to $\partial^{+} N_{i-1}$.

Definition 5.5. Given a curve $\alpha$ in a pants decomposition $P$, we define the $s u b$ surface block by the quotient

$$
B_{\alpha}=S_{\alpha} \times[0,1] /(x, t) \sim(x, 0) \quad \text { for } \quad x \in \partial S_{\alpha}, \quad t \in[0,1] .
$$

We denote the upper and lower boundary of $B_{\alpha}$ by

$$
\partial^{+} B_{\alpha}=S \times\{1\} \text { and } \partial^{-} B_{\alpha}=S \times\{0\} .
$$

We now describe block triangulations associated to each elementary move on triangulations. We will say a triangulation $\mathcal{T}$ of a subsurface block $B_{\alpha}$ realizes an elementary move $T \rightarrow \mathcal{M}(T)$ if we have

$$
\mathcal{T} \cap \partial^{-} B_{\alpha}=T \text { and } \mathcal{T} \cap \partial^{+} B_{\alpha}=\mathcal{M}(T) .
$$

Block triangulations. Let $T$ be a standard triangulation on $S_{\alpha}$ suited to $\alpha$. Then the standard block triangulation $\mathcal{T}_{\alpha}$ is obtained from

$$
T \times[0,1] / \sim
$$

in the following way. Initially, $T \times[0,1] / \sim$ is a cell decomposition of $B_{\alpha}$. For any edge $e$ of $T$ with $\partial e \cap \partial S_{\alpha}=\varnothing, e \times[0,1]$ is a quadrilateral to which we add a diagonal depending on the genus of $S_{\alpha}$.

- When $S_{\alpha}$ has genus 0 , the $\alpha$-edges $e_{\alpha}$ and $\bar{e}_{\alpha}$ determine quadrilaterals $e_{\alpha} \times[0,1]$ and $\bar{e}_{\alpha} \times[0,1]$ in $\mathcal{T}$. We triangulate these quadrilaterals with two new edges that run in the same direction along the annulus

$$
e_{\alpha} \times[0,1] \cup \bar{e}_{\alpha} \times[0,1]
$$



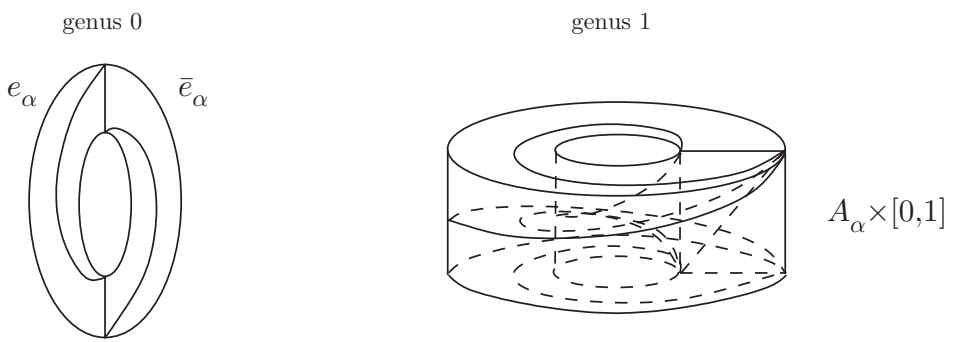

FIGURE 10. Extending $T \times[0,1] / \sim$ to a triangulation.

and cone off the new edges down to the vertices opposite the quadrilaterals (see Figure 10).

- When $S_{\alpha}$ has genus 1 , in addition to the two edges $e_{\alpha}$ and $\bar{e}_{\alpha}$ that concatenated give $\alpha$ there are 3 other edges $e_{1}, e_{2}$ and $e_{3}$ that triangulate the $\alpha$-compressing annulus $A_{\alpha}$ for which $\partial e_{j} \cap \partial S_{\alpha}=\varnothing, j=1,2,3$. We triangulate the annulus

$$
e_{\alpha} \times[0,1] \cup \bar{e}_{\alpha} \times[0,1]
$$

as before, and extend this to a triangulation of $A_{\alpha} \times[0,1]$ with no new vertices as in Figure 10.

From the standard block triangulation, we build four types of blocks:

$\boldsymbol{B L I}$. The Dehn twist block. Given $S_{\alpha}$, the block triangulation $\mathcal{T}\left(T W_{\alpha}\right)$ realizing the move $T W_{\alpha}$ is obtained from the standard block triangulation $\mathcal{T}_{\alpha}$ as follows. Consider the annulus $A=\alpha \times[0,1]$ in $B_{\alpha}$ with the triangulation $T_{A}$ on $A$ induced by $\mathcal{T}_{\alpha}$. The reference orientation for $\alpha$ locally determines a left and right side of $\alpha$ in $S_{\alpha}$, and hence a left and right side of $A$ in $B_{\alpha}$. Cut $B_{\alpha}$ along $A$ to obtain two annuli $A_{L}$ and $A_{R}$ that bound the local left and right side of $B_{\alpha}-A$. Reglue the $\alpha \times\{0\}$ boundary components of $A_{L}$ and $A_{R}$ by the identity, and reglue the $\alpha \times\{1\}$ boundary components of $A_{L}$ and $A_{R}$ shifted by a right Dehn twist.

The triangulations induced by $T_{A}$ on $A_{L}$ and $A_{R}$ determine a triangulation of the torus $A_{L} \cup A_{R}$ after regluing. This triangulation naturally extends to a triangulation of a solid torus $V$ with boundary $A_{L} \cup A_{R}$ by filling in tetrahedra (the triangulations on $A_{L}$ and $A_{R}$ differ by two pairs of diagonal switches; see Figure 11). After filling in by $V$, the result is a standard block $B_{\alpha}$ with triangulation $\mathcal{T}_{\alpha}^{\mathrm{tw}+}$ realizing the move $T W_{\alpha}$. We call the standard block $B_{\alpha}$ equipped with the triangulation $\mathcal{T}_{\alpha}^{\text {tw }+}$ the right Dehn twist block $B_{\alpha}^{\mathrm{tw}+}$.

The left Dehn twist block $B_{\alpha}^{\mathrm{tw}-}$ is obtained analogously, by regluing with a left Dehn twist instead of a right Dehn twist. The left Dehn twist block $B_{\alpha}^{\text {tw- }}$ carries the triangulation $\mathcal{T}_{\alpha}^{\text {tw }}-$ and realizes the move $T W_{\alpha}^{-1}$.

Blocks realizing the other moves are triangulated as follows.

BLII. Blow-up and blow-down blocks. We modify the standard block triangulation to obtain triangulated blocks that realize blow-up and blow-down moves as follows. To realize a genus $1 \alpha$-blow-down by a block triangulation, we modify the standard block triangulation $\mathcal{T}_{\alpha}$ on $B_{\alpha}$ by collapsing the compression annulus on $\partial^{+} B_{\alpha}$ as in the description of the move $B D_{\alpha}^{1}$. The only difference is that here in addition to collapsing triangles to edges we also collapse tetrahedra to triangles. 


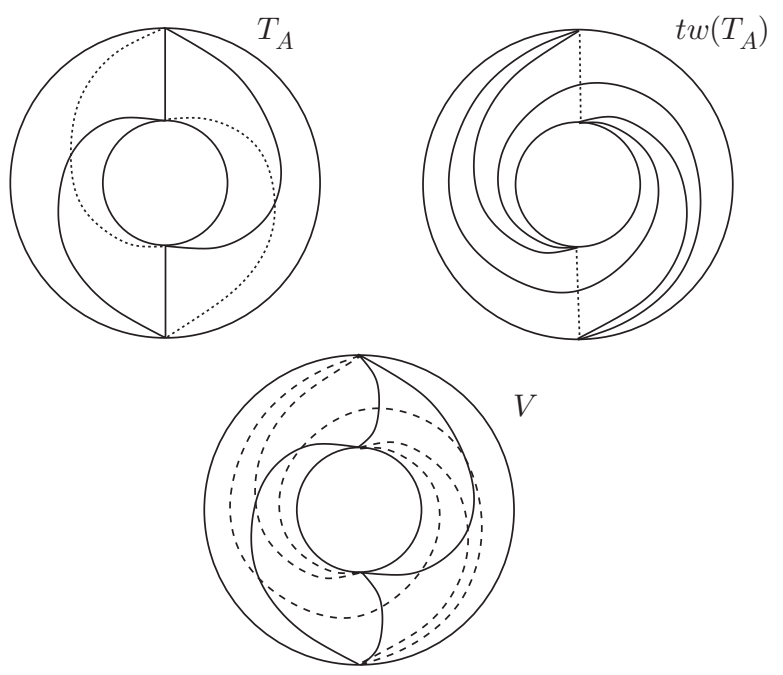

Figure 11. Triangulating the Dehn twist block. The dotted edges indicate diagonal switches for $T_{A}$ and $t w\left(T_{A}\right)$ that produce a common triangulation of the annulus, which results in the pictured triangulation of a solid torus $V$.

Similarly, if $\beta=\beta(\alpha, T)$, we obtain a block triangulation realizing the genus 1 $\beta$-blow-up $B U_{\beta}^{1}$ by collapsing the $\beta$ compression annulus on $\partial^{-} B_{\beta}$.

The genus $0 \alpha$-blow-down (and blow-up) moves come from diagonal switches on hexagons. We realize the move $B D_{\alpha}^{0}$ by gluing tetrahedra realizing each of the diagonal switches to $\partial^{+} B_{\alpha}$. Likewise, the block triangulation realizing the genus 0 $\beta$-blow-up can be obtained by gluing tetrahedra in this way to the $\beta$-hexagons on $\partial^{-} B_{\alpha}$.

We denote the $\alpha$-blow-up block by $B_{\alpha}^{\text {bu }}$ and the $\alpha$-blow-down block by $B_{\alpha}^{\text {bd }}$.

BLIII. Straightening blocks. There are two other types of block triangulations we will need that realize the identity move on a blown-down triangulation. We call these triangulated blocks straightening blocks. Given the triangulation $B D_{\alpha}^{1}(T)$ or $B D_{\alpha}^{0}(T)$ for a standard triangulation $T$ suited to a pants decomposition $P$ containing $\alpha$, the genus $g$ straightening triangulation, $g=0,1$, is obtained by completing the cell decomposition

$$
\left(B D_{\alpha}^{g}(T) \cap S_{\alpha}\right) \times[0,1] / \sim
$$

of $B_{\alpha}$, and extending this decomposition to a triangulation of $B_{\alpha}$ with no new vertices. It is easy to check that this can be done. We denote the straightening blocks by $B_{\alpha, \beta}^{\text {st }}$, where $\beta=\beta(\alpha, T)$.

5.3. Mapping in blocks and building the model. We now use our block triangulations to build a model manifold $N \cong S \times I$. We build $N$ in stages corresponding to standard triangulations $T_{j}$ suited to pants decompositions $P_{j}$ that intervene between $P_{X}$ and $P_{Y}$ respectively. At each stage there is a model $N_{j}$ also homeomorphic to $S \times I$ so that the top boundary component $\partial^{+} N_{j}=S \times\{1\}$ is triangulated by the $j$ th triangulation $T_{j}$ in the sequence of triangulations. Each $N_{j}$ will be obtained from $N_{j-1}$ by attaching a triangulated block to $\partial^{+} N_{j-1}$ that realizes the elementary move needed to move from $T_{j-1}$ to $T_{j}$. 
The model $N$ will come equipped with a map $f: N \rightarrow C(X, Y)$ that is simplicial on each block:

Definition 5.6. An incompressible mapping $f: N \rightarrow M$ from a triangulated 3manifold to a hyperbolic 3-manifold is simplicial if the lift $\widetilde{f}: \widetilde{N} \rightarrow \mathbb{H}^{3}$ sends each $k$-simplex to the convex hull of its vertices.

The following theorem describes the properties of our model and its mapping to the quasi-Fuchsian manifold $Q(X, Y)$. For simplicity we assume for the remainder of this section that $S$ is closed, and detail the necessary modifications to the argument at the end.

Theorem 5.7. Given $Q(X, Y)$, there is a model manifold $N \cong S \times I$, equipped with a surjective homotopy equivalence

$$
f: N \rightarrow \mathcal{N}_{\epsilon}(C(X, Y))
$$

to the $\epsilon$-neighborhood of the convex core $C(X, Y)$ with the following properties:

(1) $N$ is the union

$$
N=\operatorname{cap}_{X} \cup N_{\Delta} \cup \operatorname{cap}_{Y}
$$

of the caps $\operatorname{cap}_{X} \cong S \times I$ and $\operatorname{cap}_{Y} \cong S \times I$ and the triangulated part $N_{\Delta}$, a union of blocks of the above type glued top boundary to bottom boundary, all but $3 d_{\mathbf{P}}\left(P_{X}, P_{Y}\right)$ of which are Dehn twist blocks.

(2) The map $f$ is piecewise $C^{1}$ and is simplicial on $N_{\Delta}$.

(3) For each tetrahedron $\Delta$ in $N_{\Delta}$ that lies in a Dehn twist block, $f$ maps some edge of $\Delta$ to a spinning geodesic.

(4) The restriction $\left.f\right|_{\operatorname{cap}_{X}}$ is a homotopy from $\partial^{-} \mathcal{N}_{\epsilon}(C(X, Y))$ to a simplicial hyperbolic surface realizing $P_{X}$, and the restriction $\left.f\right|_{\operatorname{cap}_{Y}}$ is a homotopy from a simplicial hyperbolic surface realizing $P_{Y}$ to $\partial^{+} \mathcal{N}_{\epsilon}(C(X, Y))$.

Proof. To motivate the construction of $N$ we build the map $f$ in stages as well.

Mapping in cap $_{X}$. Let $T_{X}$ and $T_{Y}$ be standard triangulations suited to $P_{X}$ and $P_{Y}$. Let

$$
f_{X}: S \times[0,1] \rightarrow C(X, Y)
$$

determine a homotopy of the convex core boundary $g_{X}: X_{h} \rightarrow \partial^{-} C(X, Y)$ to a simplicial hyperbolic surface $h_{X}: Z_{X} \rightarrow C(X, Y)$ with associated triangulation $T_{X}$ so that if $v$ and $\bar{v}$ are the vertices of $T_{X}$ on $\alpha \in P_{X}$, we have $f_{X}(v)=p_{\alpha}$ and $f_{X}(\bar{v})=\bar{p}_{\alpha}$. Notice that this implies that $h_{X}$ realizes each curve $\alpha \in P_{X}$. Let $N_{0}=S \times[0,1]$ denote the domain for $f_{X}$; we will refer to $N_{0}=$ cap $_{X}$ as the $X$-cap of $N$. The top boundary component $\partial^{+} N_{0}$ carries the triangulation $T_{X}$.

Working inductively, we assume given a model $N_{j}$ at the $j$ th stage: i.e.

(1) the model $N_{j} \cong S \times I$ consists of the $X$-cap and a triangulated part so that the upper boundary $\partial^{+} N_{j}$ is triangulated by a standard triangulation $T_{j}$ suited to $P_{j}$;

(2) $N_{j}$ comes equipped with a map $f_{j}: N_{j} \rightarrow C(X, Y)$ that is simplicial on the triangulated part of $N_{j}$;

(3) $\left.f_{j}\right|_{\partial^{+} N_{j}}$ factors through a simplicial hyperbolic surface $h_{j}: Z_{j} \rightarrow C(X, Y)$ with associated triangulation $T_{j}$ so that $h_{j}$ sends vertices $v_{\gamma}$ and $\bar{v}_{\gamma}$ on $\gamma \in P_{j}$ to $p_{\gamma}$ and $\bar{p}_{\gamma}$ on $\gamma^{*}$. 
Let $\alpha \in P_{j}$ and $\alpha^{\prime} \in P_{j+1}$ be the curves involved in the genus $g$ elementary move $\left(P_{j}, P_{j+1}\right)$, and let $n$ be the integer guaranteed by Lemma 5.4 for which

$$
B U_{\alpha^{\prime}}^{g} \circ B D_{\alpha}^{g} \circ T W_{\alpha}^{n}\left(T_{j}\right)=T_{j+1}
$$

is a standard triangulation suited to $P_{j+1}$.

We now specify how to add triangulated blocks to $N_{j}$ and extend $f_{j}$ simplicially over each additional block to obtain the next stage of the model $N_{j+1}$.

Mapping in Dehn twist blocks. If $n$ is positive, we attach $n$ right Dehn twist blocks to the model and extend $f_{j}$ over them in sequence, while if $n$ is negative, we do likewise with left Dehn twist blocks.

We assume $n$ is positive; the negative case is identical with left Dehn twist blocks replacing right Dehn twist blocks. We attach a right Dehn twist block $B_{\alpha}^{\text {tw }}+$ to $\partial^{+} N_{j}$ so that the triangulation on $\partial^{-} B_{\alpha}^{\text {tw }}$ agrees with $T_{j} \cap S_{\alpha}$ to obtain a new model $N_{j, 1}$. We extend $f_{j}$ over $B_{\alpha}^{\mathrm{tw}+}$ to obtain a map $f_{j, 1}$ as follows. Recalling that $B_{\alpha}^{\mathrm{tw}+}$ has the form $S_{\alpha} \times[0,1] / \sim$, we set $f_{j}(x, t)=f_{j}(x, 0)$. We then straighten $f_{j}$ on $\alpha \times\{1\}$ to its geodesic representative so that the vertices $v$ and $\bar{v}$ on $\alpha \times\{1\}$ map to $p_{\alpha}$ and $\bar{p}_{\alpha}$, and finally we straighten $f_{j, 1}$ by a homotopy to make it simplicial on $B_{\alpha}^{\mathrm{tw}+}$. We note that every tetrahedron in the Dehn twist block has an edge that maps to a geodesic arc of $\alpha^{*}$, verifying part (3) in the statement of the theorem.

The map $f_{j, 1}$ factors through a simplicial hyperbolic surface still realizing $P_{j}$ with associated triangulation $T W_{\alpha}\left(T_{j}\right)$, which we denote by $T_{j, 1}$. Repeating this procedure to add $n$ Dehn twist blocks we arrive at a model $N_{j, n}$ equipped with a map $f_{j, n}: N_{j, n} \rightarrow C(X, Y)$ so that

(1) $\partial^{+} N_{j, n}$ carries the triangulation

$$
T_{j, n}=T W_{\alpha}^{n}\left(T_{j}\right),
$$

(2) $f_{j, n}$ factors through a simplicial hyperbolic surface realizing $P_{j}$ with associated triangulation $T_{j, n}$, and

(3) the vertices of $T_{j, n}$ map to $p_{\gamma}$ and $\bar{p}_{\gamma}$ on $\gamma^{*}$ for each $\gamma \in P_{j}$.

Mapping in blow-down blocks. Our discussion of how to attach a blow-down block to $N_{j, n}$ and how to extend $f_{j, n}$ over this block breaks into cases as usual.

Genus 0 . The genus 0 blow-down block $B_{\alpha}^{\text {bd }}$ is attached to $\partial^{+} N_{j, n}$ along $S_{\alpha}$ so that the triangulations agree as before. This gives a new model $N_{j, \text { bd }}$. We extend $f_{j, n}$ over $B_{\alpha}^{\text {bd }}$ to give a map $f_{j, \mathrm{bd}}: N_{j, \mathrm{bd}} \rightarrow C(X, Y)$ by first mapping $B_{\alpha}^{\mathrm{bd}}$ to $\partial^{+} N_{j, n}$, as with the Dehn twist block, and then straightening $f_{j, \text { bd }}$ to a simplicial map.

Genus 1. In the genus 1 case the construction is the same, except that as there is only one vertex $v$ on $\alpha$ in $\partial^{+} B_{\alpha}^{\mathrm{bd}}$, we simply send $v$ to $p_{\alpha}$ and straighten $f_{j \text {,bd }}$ to a simplicial map as before.

In each case the resulting map $\left.f_{j, \mathrm{bd}}\right|_{\partial^{+} N_{j, \mathrm{bd}}}$ factors through a simplicial hyperbolic surface that realizes $P_{j}$ and has associated triangulation $B D_{\alpha}^{0}\left(T_{j, n}\right)$, which we denote by $T_{j, \text { bd }}$.

Mapping in straightening blocks. Straightening blocks allow us to pass from simplicial hyperbolic surfaces realizing $P_{j}$ to simplicial hyperbolic surfaces realizing $P_{j+1}$. We attach the straightening block $B_{\alpha, \alpha^{\prime}}^{\text {st }}$ to $N_{j, \text { bd }}$ to obtain a model $N_{j, \text { st }}$. We extend $f_{j, \text { bd }}$ over $B_{\alpha, \alpha^{\prime}}^{\text {st }}$ to a map $f_{j, \text { st }}$ by defining $f_{j, \text { st }}(x, t)=f_{j, \text { st }}(x, 0)$, and then straightening $f_{j, \mathrm{st}}$ on $\alpha^{\prime} \times\{1\} \subset \partial^{+} B_{\alpha, \alpha^{\prime}}^{\text {st }}$. In the genus 1 case we send the $\alpha^{\prime}$ vertex to $p_{\alpha^{\prime}}$, and in the genus 2 case we send the $\alpha^{\prime}$ vertices to $p_{\alpha^{\prime}}$ and $\bar{p}_{\alpha^{\prime}}$, and 
finally we straighten the map to a simplicial map on $B_{\alpha, \alpha^{\prime}}^{\text {st }}$. Now

$$
\left.f_{j, \mathrm{st}}\right|_{\partial^{+} N_{j, \mathrm{st}}}
$$

factors through a simplicial hyperbolic surface realizing $P_{j+1}$ with associated triangulation $T_{j \text {,bd }}$ once again.

Mapping in blow-up blocks. This procedure is essentially the inverse of attaching and mapping in the blow-down blocks.

Genus 0. Attach $B_{\alpha^{\prime}}^{\text {bu }}$ to $\partial^{+} N_{j \text {,st }}$ along $S_{\alpha^{\prime}}$ (which equals $S_{\alpha}$ ), and extend $f_{j, \text { st }}$

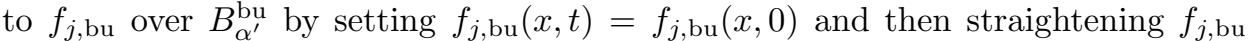
rel- $\alpha^{\prime} \times\{1\}$ to a simplicial map.

Genus 1. Set $f_{j, \mathrm{bu}}(x, t)=f_{j, \mathrm{bu}}(x, 0)$ and then homotope $f_{j, \mathrm{bu}}$ so that it still sends $\alpha^{\prime} \subset \partial^{+} B_{\alpha^{\prime}}^{\text {bu }}$ to its geodesic representative but also sends the $\alpha^{\prime}$ vertices $v_{\alpha^{\prime}}$ and $\bar{v}_{\alpha^{\prime}}$ to $p_{\alpha^{\prime}}$ and $\bar{p}_{\alpha^{\prime}}$.

The map $\left.f_{j, \text { bu }}\right|_{\partial+N_{j, \text { bu }}}$ factors through a simplicial hyperbolic surface realizing $P^{\prime}$ with associated triangulation

$$
T_{j+1}=B U_{\alpha^{\prime}} \circ B D_{\alpha} \circ T W_{\alpha}^{n}\left(T_{j}\right),
$$

which is a standard triangulation suited to $P_{j+1}$. This completes the inductive step.

Mapping in cap $_{Y}$. Let $|G|=d_{\mathbf{P}}\left(P_{X}, P_{Y}\right)$ denote the length of a geodesic $G \subset$ $\mathbf{P}(S)$. Then the above inductive procedure results finally in a map

$$
f_{|G|}: N_{|G|} \rightarrow C(X, Y)
$$

so that the restriction $\left.f_{|G|, 0}\right|_{\partial+} N_{|G|}$ factors through a simplicial hyperbolic surface realizing $P_{Y}$ with associated triangulation $T_{Y}$ (a standard triangulation suited to $\left.P_{Y}\right)$, and so that the vertices $v_{\gamma}$ and $\bar{v}_{\gamma}$ map to the vertices $p_{\gamma}$ and $\bar{p}_{\gamma}$ on the closed geodesic $\gamma^{*}$ for each $\gamma \in P_{Y}$. We complete our model $N$ by adding a $Y$-cap: this is a homotopy

$$
f_{Y}: S \times I \rightarrow C(X, Y)
$$

from $\left.f_{|G|}\right|_{\partial^{+} N_{|G|}}$ to the convex core boundary $g_{Y}: Y_{h} \rightarrow \partial^{+} C(X, Y)$. Gluing this product $S \times I$ to $\partial^{+} N_{|G|}$ and extending $f_{|G|}$ over the $Y$-cap by $f_{Y}$, we obtain the final piece of our model $N$ and the resulting map

$$
f: N \rightarrow C(X, Y),
$$

a homotopy equivalence whose restrictions

$$
\left.f\right|_{\partial^{+} N} \rightarrow \partial^{+} C(X, Y) \text { and }\left.f\right|_{\partial^{-} N} \rightarrow \partial^{-} C(X, Y)
$$

are homeomorphisms.

Though the boundary $\partial C(X, Y)$ is not generically smooth, by taking the boundary $\partial \mathcal{N}_{\epsilon}(C(X, Y))$ of the $\epsilon$-neighborhood of the convex core we obtain a pair of $C^{1}$ surfaces $\partial^{+} \mathcal{N}_{\epsilon}(C(X, Y))$ and $\partial^{-} \mathcal{N}_{\epsilon}(C(X, Y))$ with $C^{1}$ path metrics [EM] Lem. 1.3.6]. In the interest of computing volume, we perturb $f$ to a piecewise smooth map

$$
f^{\epsilon}: N \rightarrow \mathcal{N}_{\epsilon}(C(X, Y))
$$

by adjusting $f$ by a homotopy that changes $f$ only on $\operatorname{cap}_{X}$ and $\operatorname{cap}_{Y}$, so that

$$
\left.f^{\epsilon}\right|_{\partial^{+} N}: \partial^{+} N \rightarrow \partial^{+} \mathcal{N}_{\epsilon}(C(X, Y)) \text { and }\left.f^{\epsilon}\right|_{\partial^{-} N}: \partial^{-} N \rightarrow \partial^{-} \mathcal{N}_{\epsilon}(C(X, Y))
$$

are homeomorphisms, and $f^{\epsilon}$ is $C^{1}$ on the interiors of the caps of $N$. The map $f$ is already simplicial on the triangulated part of $N$ so $f^{\epsilon}$ is piecewise $C^{1}$. A degree argument shows $f^{\epsilon}$ is surjectve, proving the theorem. 
5.4. Bounding the volume. Given a piecewise differentiable 3-chain $C$ in a hyperbolic 3-manifold $M$, the function $\operatorname{deg}_{C}: M \rightarrow \mathbb{Z}$ which measures the degree of $C$ in $M$ is well defined at almost every point of $M$. We define the mass mass $(C)$ of $C$ to be the integral

$$
\operatorname{mass}(C)=\int_{M}\left|\operatorname{deg}_{C}\right| d V
$$

where $d V$ is the hyperbolic volume form on $M$ (cf. Th2, §4]).

Moreover, if $F: P \rightarrow M$ is a map of a piecewise differentiable 3-manifold $P$ to $M$, and $C$ is a piecewise differentiable 3 -chain in $P$, then we define the $F$-mass of $C$ by the integral

$$
\operatorname{mass}_{F}(C)=\int_{M}\left|\operatorname{deg}_{F(C)}\right| d V .
$$

The $F$-mass of $C$ bounds the volume $\operatorname{vol}(F(C))$ of the image of $C$ in $M$. Hence, given our piecewise differentiable surjective map

$$
f^{\epsilon}: N \rightarrow \mathcal{N}_{\epsilon}(C(X, Y)),
$$

the volume $\operatorname{vol}\left(\mathcal{N}_{\epsilon}(C(X, Y))\right.$, which bounds $\operatorname{vol}(X, Y)$, is bounded by the sums of the $f^{\epsilon}$-masses of the chains that make up $N$. In other words, if $N$ decomposes into 3 -chains $C_{k}$, we have

$$
\operatorname{vol}(X, Y) \leq \sum_{C_{k} \subset N} \operatorname{mass}_{f^{\epsilon}}\left(C_{k}\right)=: \operatorname{mass}_{f^{\epsilon}}(N)
$$

Thus, Theorem 5.1 will follow from the following proposition.

Proposition 5.8. Given $S$ there are constants $K_{3}>1$ and $K_{4}>0$ so that the map $f^{\epsilon}$ is homotopic to a map $f_{\theta}^{\epsilon}: N \rightarrow \mathcal{N}_{\epsilon}(C(X, Y))$ satisfying the conclusions of Theorem 5.7 for which

$$
\operatorname{mass}_{f_{\theta}^{\epsilon}}(N) \leq K_{3} d_{\mathbf{P}}\left(P_{X}, P_{Y}\right)+K_{4} .
$$

Let $\mathcal{V}_{3}$ denote the maximal volume of a tetrahedron in hyperbolic 3-space (see [Th1, ch. 7], [BP] $)$. We begin our approach to Proposition 5.8 with the following lemma.

Lemma 5.9. There is a constant $K_{\Delta}$ so that the map $f^{\epsilon}$ is homotopic to a map $f_{\theta}^{\epsilon}$ that also satisfies the conclusions of Theorem 5.7 so that

$$
\operatorname{mass}_{f_{\theta}^{\epsilon}}\left(N_{\Delta}\right)<K_{\Delta} \cdot \mathcal{V}_{3} \cdot d_{\mathbf{P}}\left(P_{X}, P_{Y}\right)+1 .
$$

Proof. Because of the possibility of a large number of Dehn twist blocks in $N_{\Delta}$, there is not in general a uniform constant $K$ for which the number of tetrahedra used to triangulate $N_{\Delta}$ is less than $K d_{\mathbf{P}}\left(P_{X}, P_{Y}\right)$. We will show, however, that by modifying $f^{\epsilon}$ by a homotopy, we can force the tetrahedra that lie in Dehn twist blocks to have mass as small as we like.

By Theorem 5.7, the number of blocks in $N_{\Delta}$ that are not Dehn twist blocks is bounded by $3 d_{\mathbf{P}}\left(P_{X}, P_{Y}\right)$. Since there is a uniform bound to the number of tetrahedra in any block, there is a constant $K_{\Delta}$ so that all but at most $K_{\Delta} d_{\mathbf{P}}\left(P_{X}, P_{Y}\right)$ tetrahedra of $N_{\Delta}$ lie in Dehn twist blocks.

Let $\alpha^{*} \in \operatorname{spin}(G)$ be a spinning geodesic. Given $\theta \in \mathbb{R}$, let $f_{\theta}^{\epsilon}$ be defined by the following homotopy of $f^{\epsilon}$ through maps that are simplicial on $N_{\Delta}$ : for each $\alpha \in \bigcup_{j} P_{j}$, slide the vertices $p_{\alpha}$ and $\bar{p}_{\alpha}$ along the geodesic $\alpha^{*}$ a distance

$$
\frac{\theta}{2 \pi} \ell_{Q(X, Y)}\left(\alpha^{*}\right)
$$




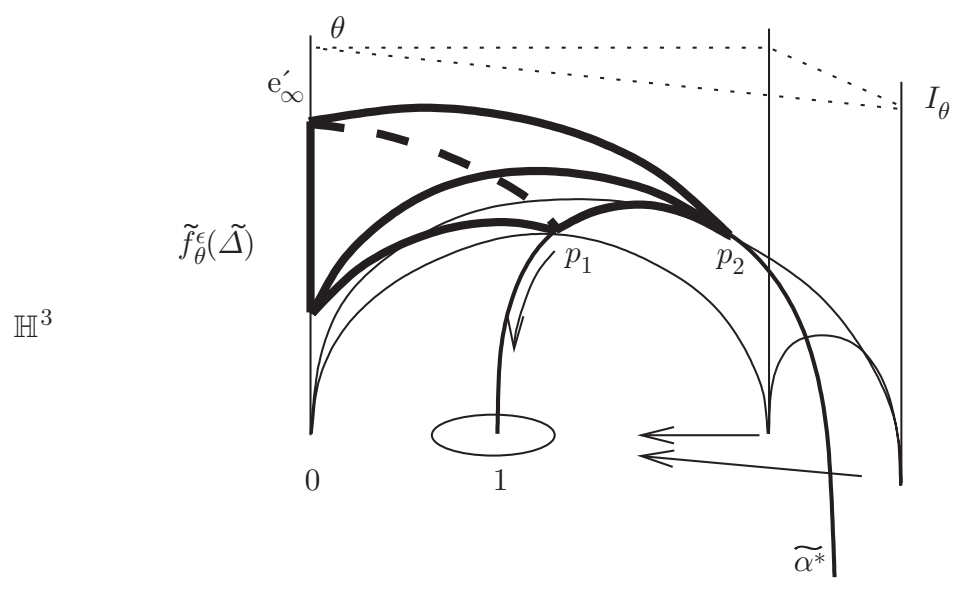

Figure 12. Spinning tetrahedra in $\mathbb{H}^{3}$.

in the direction of the reference orientation chosen for $\alpha$. (See [Th2] for another example of this spinning of triangulations.) The following lemma shows that tetrahedra that lie in a Dehn twist block, which we will call Dehn twist tetrahedra, can be made to have small $f_{\theta}^{\epsilon}$-mass by spinning to sufficiently high values of $\theta$.

Lemma 5.10. If $\Delta \subset N_{\Delta}$ is a Dehn twist tetrahedron, then

$$
\operatorname{mass}_{f_{\theta}^{\epsilon}}(\Delta) \rightarrow 0 \quad \text { as } \quad \theta \rightarrow \infty
$$

Proof. Recall from Theorem 5.7 each Dehn twist tetrahedron $\Delta$ has at least one edge $e$ for which $f^{\epsilon}(e) \subset \alpha^{*}$ for some spinning geodesic $\alpha^{*}$.

Lift $f_{\theta}^{\epsilon}$ to $\widetilde{f_{\theta}^{\epsilon}}: \widetilde{N} \rightarrow \widetilde{\mathbb{H}^{3}}$ and choose a lift $\widetilde{\Delta}$ to $\widetilde{N}$. Let $\widetilde{\alpha^{*}}$ denote the lift of $\alpha^{*}$ to $\mathbb{H}^{3}$ for which $\widetilde{f_{\theta}^{\epsilon}}$ sends the lifted edge $\widetilde{e} \subset \widetilde{\Delta}$ to $\widetilde{\alpha^{*}}$. Let $e^{\prime}$ be the opposite edge of $\Delta$ (e and $e^{\prime}$ have no endpoints in common).

Let $I_{\theta}$ be the ideal tetrahedron in $\mathbb{H}^{3}$ for which

(1) $\widetilde{f_{\theta}^{\epsilon}}\left(\widetilde{e^{\prime}}\right)$ lies in one edge $e_{\infty}^{\prime}$ of $I_{\theta}$;

(2) the two other edges $e_{\infty}^{1}$ and $e_{\infty}^{2}$ of $I_{\theta}$ emanating from one endpoint of $e_{\infty}^{\prime}$ pass through endpoints $p_{1}$ and $p_{2}$ of $\widetilde{f}_{\theta}^{\epsilon}(\widetilde{e})$.

The image $\widetilde{f_{\theta}^{\epsilon}}(\widetilde{\Delta})$ lies in $I_{\theta}$ (see Figure 12 ).

Normalize by an isometry of $\mathbb{H}^{3}$ so that the edge $e_{\infty}^{\prime}$ of $I_{\theta}$ has its ideal endpoints at 0 and $\infty$, and so that the terminal fixed point of $\widetilde{\alpha^{*}}$ (with respect to the reference orientation for $\alpha$ ) lies at $1 \in \mathbb{C}$.

Then for any $r>0$ there exists a $\theta$ so that the two ideal vertices of $I_{\theta}$ that do not lie at 0 and $\infty$ lie within a small disk $|1-z|<r$. It follows that the dihedral angle of $I_{\theta}$ at $e_{\infty}^{\prime}$ tends to 0 as $\theta \rightarrow \infty$. But the volume of an ideal tetrahedron tends to 0 as any of its dihedral angles tends to 0 , so we have

$$
\operatorname{mass}_{f_{\theta}^{\epsilon}}(\Delta)<\operatorname{vol}\left(I_{\theta}\right) \rightarrow 0 \quad \text { as } \quad \theta \rightarrow \infty .
$$

Continuation of the proof of Lemma 5.9: By Lemma 5.10, for any Dehn twist block $B$, the quantity $\operatorname{mass}_{f_{\theta}^{\epsilon}}(B)$ is as small as we like for $\theta$ sufficiently large. If $\mathcal{B}^{\text {tw }}$ 
denotes the union of all Dehn twist blocks in $N_{\Delta}$, then we may choose $\theta$ sufficiently large so that

$$
\operatorname{mass}_{f_{\theta}^{\epsilon}}\left(\mathcal{B}^{\mathrm{tw}}\right)<1
$$

Since $f_{\theta}^{\epsilon}$ is simplicial on $N_{\Delta}$, we have

$$
\operatorname{mass}_{f_{\theta}^{\epsilon}}(\Delta)<\mathcal{V}_{3}
$$

for any tetrahedron $\Delta \subset N_{\Delta}$, and since all but at most $K_{\Delta} d_{\mathbf{P}}\left(P_{X}, P_{Y}\right)$ tetrahedra in $N_{\Delta}$ lie in $\mathcal{B}^{\text {tw }}$, we have

$$
\operatorname{mass}_{f_{\theta}^{\epsilon}}\left(N_{\Delta}\right)<K_{\Delta} \cdot \mathcal{V}_{3} \cdot d_{\mathbf{P}}\left(P_{X}, P_{Y}\right)+1 .
$$

Bounding the volume of the caps. The bound on the $f_{\theta}^{\epsilon}$-mass of the triangulated part $N_{\Delta}$ in terms of the distance $d_{\mathbf{P}}\left(P_{X}, P_{Y}\right)$ will be sufficient for Proposition 5.8 once we show the following uniform bound on the $f_{\theta}^{\epsilon}$-mass of the caps of $N$.

Lemma 5.11. There is a uniform constant $K_{\text {cap }}$, depending only on $S$ so that after modifying $f_{\theta}^{\epsilon}$ by a homotopy on $\operatorname{cap}_{X}$ we have

$$
\operatorname{mass}_{f_{\theta}^{\epsilon}}\left(\operatorname{cap}_{X}\right)<K_{\text {cap }}
$$

and similarly for $\operatorname{cap}_{Y}$.

Proof. Our goal will be to modify the homotopy $\left.f_{\theta}^{\epsilon}\right|_{\operatorname{cap}_{X}}$ from $\partial^{-} \mathcal{N}_{\epsilon}(C(X, Y))$ to the simplicial hyperbolic surface $Z_{X}$ by cutting the surface $S$ into annuli and controlling the trace of the homotopy on each annulus (a solid torus). To obtain such control, we choose this decomposition compatibly with the pants decomposition $P_{X}$.

We fix attention on a single pair of pants $\widehat{S} \subset S-P_{X}$. By a figure- 8 curve on $\widehat{S}$ we will mean a closed curve that intersects itself once on $\widehat{S}$ and divides $\widehat{S}$ into three annuli, one parallel to each boundary component of $\widehat{S}$ (see Figure 13). To prove Lemma 5.11 we establish the following basic lemma in hyperbolic surface geometry. (We continue to treat the closed case; the case when $S$ has boundary is similar.)

Lemma 5.12. Let $S$ a closed surface with negative Euler characteristic, and let $L^{\prime} \geq L$ be a constant greater than or equal to the Bers constant $L$ for $S$. Then there is a constant $L_{8}\left(L^{\prime}\right)>0$ so that the following holds: if $P$ is a pants decomposition of $S, Z \in \operatorname{Sing}_{k}(S)$ is a possibly singular hyperbolic surface, and the length bound $\ell_{Z}(\alpha)<L^{\prime}$ holds for each $\alpha \in P$, then any figure-8 curve $\gamma$ in any component $\widehat{S} \subset S-P$ satisfies

$$
\ell_{Z}(\gamma)<L_{8}\left(L^{\prime}\right)
$$

Proof. Note that any bound on the geodesic length of a given figure- 8 curve $\gamma$ guarantees a bound on the geodesic length of any other, by taking twice the original bound.

Assume first that $Z \in \operatorname{Teich}(S)$, so $Z$ is a non-singular hyperbolic surface. From the thick-thin decomposition for hyperbolic surfaces, given any $\delta>0$, there is a uniform bound $D_{\delta}$ to the diameter $\operatorname{diam}\left(Z_{\geq \delta}\right)$ of the $\delta$-thick part of $Z$, where $D_{\delta}$ depends only on $\delta$ and the surface $S$. Let $\partial \widehat{S}=\alpha_{1} \sqcup \alpha_{2} \sqcup \alpha_{3}$. Since $\ell_{Z}\left(\alpha_{i}\right)<L^{\prime}$, there is a $\delta$ so that $\alpha_{i}^{*}$ can only intersect $Z_{<\delta}$ in a component of $Z_{<\delta}$ for which it is the core curve. Choosing $\delta$ smaller if necessary, we can ensure that the boundary of any component of $Z_{<\delta}$ is a curve of length less than $L^{\prime}$. 


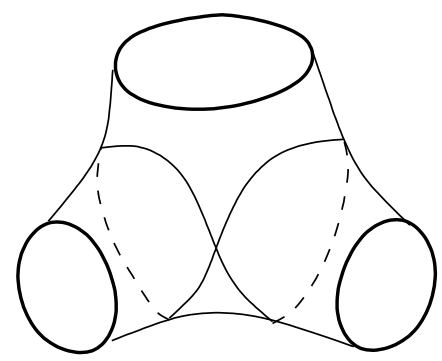

Figure 13. A figure eight curve.

Let $\widehat{Z}$ be the realization of $\widehat{S}$ as a subsurface of $Z$ bounded by the curves $\bigcup \alpha_{i}^{*}$. Either two of the $\alpha_{i}^{*}$ lie entirely in $Z_{\geq \delta}$ or two of the $\alpha_{i}$ are homotopic into $Z_{<\delta}$. Without loss of generality, assume $\alpha_{1}^{*}$ and $\alpha_{2}^{*}$ lie in $Z_{\geq \delta}$. Then they can be joined by an oriented arc $b$ in $\widehat{Z}$ of length less than $D_{\delta}$. Either $\alpha_{1} \cdot b \cdot \alpha_{2} \cdot b^{-1}$ or $\alpha_{1} \cdot b \cdot \alpha_{2}^{-1} \cdot b^{-1}$ is a figure- 8 curve of length less than $2 L^{\prime}+2 D_{\delta}$. Otherwise, if $A_{1}$ and $A_{2}$ are components of $Z_{<\delta}$ representing the homotopy classes of $\alpha_{1}$ and $\alpha_{2}$, then there are components $a_{1}$ of $\partial A_{1}$ and $a_{2}$ of $\partial A_{2}$ with length less than $L^{\prime}$, and an oriented arc $b$ joining $a_{1}$ to $a_{2}$ in $\widehat{Z}$ of length less than $D_{\delta}$. By the same reasoning, there is a figure- 8 curve $\gamma$ of length

$$
\ell_{Z}(\gamma)<2 L^{\prime}+2 D_{\delta}
$$

To treat the potentially singular case, let $Z \in \operatorname{Sing}_{k}(S)$ satisfy $\ell_{Z}\left(\alpha_{i}\right)<L^{\prime}$ for each $\alpha_{i} \in P$. Let $Z^{\mathrm{h}} \in \operatorname{Teich}(S)$ represent the hyperbolic surface in the same conformal class as $Z$. If each $\alpha_{i} \in P$ satisfies

$$
\ell_{Z^{\mathrm{h}}}\left(\alpha_{i}\right)<L^{\prime}
$$

then there is a figure- 8 curve $\gamma \subset \widehat{S}$ with $\ell_{Z^{\mathrm{h}}}(\gamma)<2 L^{\prime}+2 D_{\delta}$ by the above reasoning. By Ahlfors' lemma Ah we have

$$
\ell_{Z}(\gamma)<\ell_{Z^{\mathrm{h}}}(\gamma)<2 L^{\prime}+2 D_{\delta}
$$

proving the lemma in this case.

If, however, some $\alpha_{i} \subset \partial \widehat{S}$ satisfies $\ell_{Z^{\mathrm{h}}}\left(\alpha_{i}\right) \geq L^{\prime} \geq L$, then by Theorem 2.1 there is a simple closed curve $\beta$, with $i\left(\alpha_{i}, \beta\right) \neq 0$, for which $\ell_{Z^{\mathrm{h}}}(\beta)<L$. Again applying Ahlfors' lemma, the curve $\beta$ has length

$$
\ell_{Z}(\beta)<L
$$

on $Z$, so its geodesic representative $\beta^{*}$ furnishes an $\operatorname{arc} b$ in $\widehat{S}$ that joins $\alpha_{i}^{*}$ to $\alpha_{j}^{*}$, or joins $\alpha_{i}^{*}$ to itself. In either case, two copies of $b$ together with $\operatorname{arcs}$ in the geodesics at its endpoints can be assembled to form a figure- 8 curve $\beta \subset \widehat{S}$ with length bounded by

$$
\ell_{Z}(\beta)<2 L^{\prime}+2 L \leq 4 L^{\prime}
$$

By setting

the lemma follows.

$$
L_{8}\left(L^{\prime}\right)=4 L^{\prime}+2 D_{\delta}
$$

Remark. Though we continue to work in the setting where $S$ is closed, the proof of the lemma for the case when $\partial S \neq \varnothing$ goes through simply. 
Continuation of the proof of Lemma 5.11: Again consider the subsurface $\widehat{S}$ and its realization as a subsurface $\widehat{Z_{X}} \subset Z_{X}$ bounded by geodesics $\hat{\alpha_{1}}, \hat{\alpha_{2}}$ and $\hat{\alpha_{3}}$ on $Z_{X}$. Let

$$
X_{h}^{\epsilon}=\partial^{+} \mathcal{N}_{\epsilon}(C(X, Y))
$$

denote the boundary component of $\mathcal{N}_{\epsilon}(C(X, Y))$ facing $X$, and let $\widehat{X_{h}^{\epsilon}}$ denote the realization of $\widehat{S}$ as a subsurface $X_{h}^{\epsilon}$ bounded by geodesics $\overline{\alpha_{1}}, \overline{\alpha_{2}}$ and $\overline{\alpha_{3}}$ in the path metric on $X_{h}^{\epsilon}$. Let $\gamma$ be a figure- 8 curve on $\widehat{S}$ with geodesic representatives $\hat{\gamma}$ and $\bar{\gamma}$ on $\widehat{Z_{X}}$ and $\widehat{X_{h}^{\epsilon}}$ respectively.

Let $A_{1}, A_{2}$ and $A_{3}$ denote the three annuli in $\widehat{S}-\gamma$ for which $\alpha_{i} \subset \partial A_{i}$, where $i=1,2,3$, and let $\gamma_{i} \subset \gamma$ be the simple loop in $\gamma$ so that $\gamma_{i} \subset \partial A_{i}$ for $i=1,2,3$. Let $\hat{A}_{i}$ be the realization of $A_{i}$ on $\widehat{Z_{X}}$ with $\partial \hat{A_{i}} \subset \hat{\alpha_{i}} \cup \hat{\gamma}$, and let $\bar{A}_{i}$ be the realization of $A_{i}$ on $\widehat{X_{h}^{\epsilon}}$ with $\partial \bar{A}_{i} \subset \bar{\alpha}_{i} \cup \bar{\gamma}$.

By a theorem of D. Sullivan [Sul1] elaborated upon by D. Epstein and A. Marden [EM, Thm. 2.3.1] the path metric on the surface $X_{h}^{\epsilon}$ has bounded distortion from the hyperbolic metric on $X$ : the nearest point retraction map (see [EM]) is $4 \cosh (\epsilon)$ Lipschitz, in particular. It follows that there is a constant $C_{5}>4$ so that choosing $\epsilon$ sufficiently small we have

$$
\ell_{X_{h}^{\epsilon}}\left(\bar{\alpha}_{i}\right)<C_{5} L
$$

and

$$
\operatorname{area}\left(\widehat{X_{h}^{\epsilon}}\right)<\operatorname{area}\left(X_{h}^{\epsilon}\right)<C_{5} 2 \pi|\chi(S)|
$$

where $i=1,2,3$. Since $\widehat{Z_{X}}$ is triangulated by 8 hyperbolic triangles, we have

$$
\operatorname{area}\left(\hat{A}_{i}\right)<8 \pi
$$

for $i=1,2,3$.

Returning to the map $f_{\theta}^{\epsilon}: N \rightarrow \mathcal{N}_{\epsilon}(C(X, Y))$, we may define the $f_{\theta}^{\epsilon}$-mass of a piecewise differentiable 2-cycle in $N$ similarly to that of a 3 -chain, by integrating the absolute value of the degree with respect to two-dimensional Lebesgue measure on $M$ (cf. [Th2 Prop. 4.1] and the preceding discussion).

We modify the map $f_{\theta}^{\epsilon}$ on cap $_{X} \cong S \times I$ as follows. By Theorem 5.7 the map $f_{\theta}^{\epsilon}$ already sends $\alpha_{i} \times 0$ to the geodesic $\hat{\alpha_{i}}$ for each $\alpha_{i} \in P_{X}$. Straighten $f_{\theta}^{\epsilon}$ without changing its values on $\partial^{+} \operatorname{cap}_{X}$ so that $f_{\theta}^{\epsilon}$ sends each $\alpha_{i} \times\{0\}$ to the geodesic $\bar{\alpha}_{i} \subset X_{h}^{\epsilon}$, and so that $f_{\theta}^{\epsilon}$ sends each track $x \times[0,1]$, where $x$ lies in $\alpha_{i}$, to a geodesic. The image $f_{\theta}^{\epsilon}\left(\alpha_{i} \times[0,1]\right)$ is a ruled annulus, and any ruled annulus has area less than the sum of the lengths of its boundary components (see e.g. [Th1, Ch. 9], [Bon, $\S 3.2])$. Since $\ell_{X_{h}^{\epsilon}}\left(\overline{\alpha_{i}}\right)<C_{5} L$, and since $\hat{\alpha_{i}}$ is the geodesic representative of $\alpha_{i}$ in $Q(X, Y)$, we have

$$
\operatorname{mass}_{f_{\theta}^{\epsilon}}\left(\alpha_{i} \times[0,1]\right)<2 C_{5} L .
$$

Applying Lemma 5.12 to $X$ with $L^{\prime}=L$, we have an $L_{8}=L_{8}(L)$ so that

$$
\ell_{X}(\gamma)<L_{8}
$$

It follows that

$$
\ell_{X_{h}^{\epsilon}}(\gamma)<C_{5} L_{8}
$$

Applying Lemma 5.12 to $Z_{X}$ with $L^{\prime}=C_{5} L$, we have an $L_{8}^{\prime}=L_{8}\left(C_{5} L\right)$ for which

$$
\ell_{Z_{X}}(\gamma)<L_{8}^{\prime} .
$$

Letting

$$
K_{8}=\max \left\{L_{8}, L_{8}^{\prime}\right\},
$$


we may straighten $f_{\theta}^{\epsilon}$ to send $\gamma \times\{0\}$ to its geodesic representative $\bar{\gamma} \subset X_{h}^{\epsilon}$ in $X_{h}^{\epsilon}$ and straighten further as before so that for $x \in \gamma$ the map $f_{\theta}^{\epsilon}$ sends $x \times[0,1]$ to a geodesic. Arguing as for $\alpha_{i}$, each annulus $\gamma_{i} \times[0,1]$ has mass

$$
\operatorname{mass}_{f_{\theta}^{\epsilon}}\left(\gamma_{i} \times[0,1]\right)<2 K_{8}
$$

It follows that the union of the four annuli

$$
\mathcal{T}_{i}=\left(A_{i} \times\{1\}\right) \cup\left(\alpha_{i} \times[0,1]\right) \cup\left(A_{i} \times\{0\}\right) \cup\left(\gamma_{i} \times[0,1]\right)
$$

along their boundaries is a torus with mass

$$
\operatorname{mass}_{f_{\theta}^{\epsilon}}\left(\mathcal{T}_{i}\right)<2 K_{8}+(8+2|\chi(S)|) \pi=K_{8}^{\prime} .
$$

Applying [Th2, Prop. 4.1], we have that the solid torus $\mathcal{V}_{i} \subset N$ bounded by $\mathcal{T}_{i}$ has mass

$$
\operatorname{mass}_{f_{\theta}^{\epsilon}}\left(\mathcal{V}_{i}\right) \leq \operatorname{mass}_{f_{\theta}^{\epsilon}}\left(\mathcal{T}_{i}\right)<K_{8}^{\prime}
$$

It follows that the total $f_{\theta}^{\epsilon}$-mass of $\widehat{S} \times I$ is less than $3 K_{8}^{\prime}$, and since the number of pieces of the complement $S-P_{X}$ depends only on $S$, it follows that

$$
\operatorname{mass}_{f_{\theta}^{\epsilon}}\left(\operatorname{cap}_{X}\right)<K_{\text {cap }}
$$

for an a priori constant $K_{\text {cap }}$.

Conclusion. The proof of Proposition 5.8 is now an application of the preceding lemmas.

Proof of Proposition 5.8. Applying Lemmas 5.9 and 5.11, we have

$$
\begin{aligned}
\operatorname{mass}_{f_{\theta}^{\epsilon}}(N) & =\operatorname{mass}_{f_{\theta}^{\epsilon}}\left(N_{\Delta}\right)+\operatorname{mass}_{f_{\theta}^{\epsilon}}\left(\operatorname{cap}_{X}\right)+\operatorname{mass}_{f_{\theta}^{\epsilon}}\left(\operatorname{cap}_{Y}\right) \\
& <K_{\Delta} \cdot \mathcal{V}_{3} \cdot d_{\mathbf{P}}\left(P_{X}, P_{Y}\right)+1+2 K_{\text {cap }} .
\end{aligned}
$$

Setting $K_{\Delta} \cdot \mathcal{V}_{3}=K_{3}$ and $1+2 K_{\text {cap }}=K_{4}$, the result follows.

The case when $\partial S \neq \varnothing$. Minor modifications to the above arguments are required when $\partial S \neq \varnothing$, and $Q(X, Y)$ has peripheral rank-1 cusps. The primary difference in our model $N$ in this case arises from the fact that boundary curves of $S$ are not elements of the pants decompositions $P_{j}$ interpolating between $P_{X}$ and $P_{Y}$. For the purposes of the construction, triangulations suited to $P_{j}$ are then triangulations of $S$ with two vertices on each boundary component so that on each pair of pants in $S-P_{j}$ the triangulation has the structure of Definition 5.3. The blocks required to accomplish each elementary move are then identical; to map them into $C(X, Y)$ we need only specify the behavior of the maps $f_{j, *}$ on the boundary curves.

Indeed, if $\gamma \subset \partial S$, there is no geodesic in the free homotopy class of $\gamma$. Choosing a horocycle $h$ in the homotopy class of $\gamma$ on the boundary $\partial\left(C(X, Y)_{\geq \epsilon_{0}}\right)$ and a pair of antipodal vertices $p_{h}$ and $\bar{p}_{h}$ on $h$, we let $\gamma^{*}$ denote the piecewise geodesic obtained from straightening $h$ rel- $p_{h} \cup \bar{p}_{h}$. The behavior of $f_{j, *}$ is then determined by requiring that $f_{j, *}$ send each $\gamma \subset \partial S$ to the corresponding $\gamma^{*}$ and that $f_{j, *}$ be simplicial as before.

If $f_{\Delta}: N_{\Delta} \rightarrow C(X, Y)$ denotes the simplicial map of $N_{\Delta}$ into $C(X, Y)$ given by the above instructions, we modify $f_{\Delta}$ by sending $\epsilon_{0}$ to 0 : the edges and vertices of $N_{\Delta}$ incident on $\gamma \subset \partial S$ are mapped by $f_{\Delta}$ deeper and deeper into the $\gamma$-cusp so that the limiting map $f_{\Delta, 0}$ sends each such tetrahedron to a tetrahedron or triangle with one vertex at infinity. The restriction of $f_{\Delta, 0}$ to the interiors of $\partial^{+} N_{\Delta}$ and $\partial^{-} N_{\Delta}$ factor through simplicial hyperbolic surfaces $h_{X}: Z_{X} \rightarrow C(X, Y)$ and 
$h_{Y}: Z_{Y} \rightarrow C(X, Y)$ realizing $P_{X}$ and $P_{Y}$, and the restriction of $f_{\Delta, 0}$ to $N_{\Delta}$ with the $\gamma$ curves removed is a proper homotopy from $h_{X}$ to $h_{Y}$.

The caps, now proper homotopies from $Z_{X}$ to $X_{h}^{\epsilon}$ and $Z_{Y}$ to $Y_{h}^{\epsilon}$ may then be added to the triangulated part. The total mass is again bounded by $K_{\text {cap }}$, since the caps still decompose into solid tori with boundary of bounded area (some are now bounded by properly embedded annuli asymptotic to cusps). The resulting map, a proper homotopy between the two components $X_{h}^{\epsilon}$ to $Y_{h}^{\epsilon}$ of $\partial \mathcal{N}_{\epsilon}(C(X, Y))$ may be spun about the spinning geodesics as before to force the Dehn twist tetrahedra to have arbitrarily small mass. Spinning sufficiently far, then, we again have the conclusion of Proposition 5.8

\section{Geometric limits}

We conclude with the following application of our results to the study of algebraic and geometric limits of hyperbolic 3-manifolds.

Theorem 6.1. Let $\left\{Q\left(X_{k}, Y_{k}\right)\right\}_{k=1}^{\infty} \subset A H(S)$ be an algebraically and geometrically convergent sequence with geometric limit $N_{G}$. Then $N_{G}$ is geometrically finite if and only if there is a $K>0$ for which

$$
d_{\mathrm{WP}}\left(X_{k}, Y_{k}\right)<K
$$

for all $k$.

Proof. If the geometric limit $N_{G}$ is geometrically infinite, the volumes of the convex cores of the approximates $Q\left(X_{k}, Y_{k}\right)$ grow without bound (see [CM, Lem. 7.1]), which, applying Theorem 1.2, implies that $d_{\mathrm{WP}}\left(X_{k}, Y_{k}\right)$ grows without bound.

Likewise, applying [Ta] or [Mc3] Thm. 3.1] when $N_{G}$ is geometrically finite, the $\epsilon$-thick parts of the convex cores of $Q\left(X_{k}, Y_{k}\right)$ converge geometrically to that of $N_{G}$ for $\epsilon$ sufficiently small. It follows that the volume of $\operatorname{core}\left(Q\left(X_{k}, Y_{k}\right)\right)_{\geq \epsilon}$ converges to the volume of $\operatorname{core}\left(N_{G}\right)_{\geq \epsilon}$ for each sufficiently small $\epsilon$ and thus that

$$
\operatorname{vol}\left(\operatorname{core}\left(Q\left(X_{k}, Y_{k}\right)\right)\right) \rightarrow \operatorname{vol}\left(\operatorname{core}\left(N_{G}\right)\right)
$$

as $k$ tends to $\infty$. The theorem follows.

\section{ACKNOWLEDGEMEnts}

I would like to thank Yair Minsky, in particular, for introducing me to Thurston's conjecture and for suggesting the use of curve hierarchies to improve the volume lower bound. Thanks also to Bill Thurston, Steve Kerckhoff, and Curt McMullen for useful conversations, and to Lewis Bowen and the referee for corrections and suggestions.

\section{REFERENCES}

[Ah] L. Ahlfors. An extension of Schwarz's lemma. Trans. Amer. Math. Soc. 43(1938), 359-364.

[BP] R. Benedetti and C. Petronio. Lectures on Hyperbolic Geometry. Springer-Verlag, 1992. MR 94e:57015

[Brs1] L. Bers. Simultaneous uniformization. Bull. AMS 66(1960), 94-97. MR 22:2694

[Brs2] L. Bers. On boundaries of Teichmüller spaces and on Kleinian groups: I. Annals of Math. 91(1970), 570-600. MR 45:7044

[Brs3] L. Bers. Spaces of degenerating Riemann surfaces. In Discontinuous groups and Riemann surfaces, pages 43-55. Annals of Math Studies 76, Princeton University Press, 1974. MR 50:13497 
[Bon] F. Bonahon. Bouts des variétés hyperboliques de dimension 3. Annals of Math. 124(1986), 71-158. MR 88c:57013

[Bow] R. Bowen. Hausdorff dimension of quasi-circles. Publ. Math. IHES 50(1979), 11-25. MR 81g:57023

[Br1] J. Brock. Continuity of Thurston's length function. Geom. \& Funct. Anal. 10(2000), 741797. MR 2001g:57028

[Br2] J. Brock. Iteration of mapping classes and limits of hyperbolic 3-manifolds. Invent. Math. 143(2001), 523-570. MR 2002d:30052

[Br3] J. Brock. Weil-Petersson translation distance and volumes of mapping tori. To appear, Comm. Anal. Geom.

[BF] J. Brock and B. Farb. Rank and curvature of Teichmüller space. Preprint (2001). Submitted for publication.

[BC] M. Burger and R. Canary. A lower bound on $\lambda_{0}$ for geometrically finite hyperbolic $n$ manifolds. J. Reine Angew. Math. 454(1994), 37-57. MR 95h:58138

[Bus] P. Buser. Geometry and spectra of compact Riemann surfaces. Birkhauser Boston, 1992. MR 93g:58149

[Can1] R. D. Canary. On the Laplacian and the geometry of hyperbolic 3-manifolds. J. Diff. Geom. 36(1992), 349-367. MR 93g:57016

[Can2] R. D. Canary. Ends of hyperbolic 3-manifolds. J. Amer. Math. Soc. 6(1993), 1-35. MR 93e:57019

[Can3] R. D. Canary. A covering theorem for hyperbolic 3-manifolds and its applications. Topology 35(1996), 751-778. MR 97e:57012

[CM] R. D. Canary and Y. N. Minsky. On limits of tame hyperbolic 3-manifolds. J. Diff. Geom. 43(1996), 1-41. MR 98f:57021

[EM] D. B. A. Epstein and A. Marden. Convex hulls in hyperbolic space, a theorem of Sullivan, and measured pleated surfaces. In Analytical and Geometric Aspects of Hyperbolic Space, pages 113-254. Cambridge University Press, 1987. MR 89c:52014

[Gard] F. Gardiner. Teichmüller theory and quadratic differentials. Wiley Interscience, 1987. MR 88m:32044

[Har] W. J. Harvey. Boundary structure of the modular group. In Riemann Surfaces and Related Topics: Proceedings of the 1978 Stony Brook Conference. Annals of Math Studies 97, Princeton University Press, 1981. MR 83d:32022

[Hat] A. Hatcher. On triangulations of surfaces. Topology Appl. 40(1991), 189-194. MR 92f: 57020

[HLS] A. Hatcher, P. Lochak, and L. Schneps. On the Teichmüller tower of mapping class groups. J. Reine Angew. Math. 521(2000), 1-24. MR 2001h:57018

[HT] A. Hatcher and W. Thurston. A presentation for the mapping class group. Topology 19(1980), 221-237. MR 81k:57008

[IT] Y. Imayoshi and M. Taniguchi. An introduction to Teichmüller spaces. Springer-Verlag, 1992. MR 94b:32031

[Mas] H. Masur. The extension of the Weil-Petersson metric to the boundary of Teichmüller space. Duke Math. J. 43(1976), 623-635. MR 54:5506

[MM1] H. Masur and Y. Minsky. Geometry of the complex of curves I: hyperbolicity. Invent. Math. 138(1999), 103-149. MR 2000i:57027

[MM2] H. Masur and Y. Minsky. Geometry of the complex of curves II: hierarchical structure. Geom. E Funct. Anal. 10(2000), 902-974. MR 2001k:57020

[Mc1] C. McMullen. Iteration on Teichmüller space. Invent. math. 99(1990), 425-454. MR 91a:57008

[Mc2] C. McMullen. Renormalization and 3-manifolds which fiber over the circle. Annals of Math. Studies 142, Princeton University Press, 1996. MR 97f:57022

[Mc3] C. McMullen. Hausdorff dimension and conformal dynamics I: Strong convergence of Kleinian groups. J. Diff. Geom. 51(1999), 471-515. MR 2001c:37045

[Min] Y. Minsky. The classification of punctured torus groups. Annals of Math. 149(1999), 559626. MR 2000f:30028

[Ot] J. P. Otal. Le théorème d'hyperbolisation pour les variétés fibrées de dimension trois. Astérisque, 1996. MR 97e:57013

[Sul1] D. Sullivan. Travaux de Thurston sur les groupes quasi-fuchsiens et sur les variétés hyperboliques de dimension 3 fibrées sur $S^{1}$. Sem. Bourbaki 554(1979/80). MR 83h:58079 
[Sul2] D. Sullivan. Aspects of positivity in Riemannian geometry. J. Diff. Geom. 25(1987), 327351. MR 88d:58132

[Ta] E. Taylor. Geometric finiteness and the convergence of Kleinian groups. Comm. Anal. Geom. 5(1997), 497-533. MR 98k:30064

[Th1] W. P. Thurston. Geometry and topology of three-manifolds. Princeton lecture notes, 1979.

[Th2] W. P. Thurston. Hyperbolic structures on 3-manifolds I: Deformations of acylindrical manifolds. Annals of Math. 124(1986), 203-246. MR 88g:57014

[Tro] A. J. Tromba. On a natural algebraic affine connection on the space of almost complex structures and the curvature of Teichmüller space with respect to its Weil-Petersson metric. Manuscripta Math. 56(1986), 475-497. MR 88c:32034

[Wol1] S. Wolpert. Noncompleteness of the Weil-Petersson metric for Teichmüller space. Pacific J. Math. 61(1975), 573-577. MR 54:10678

[Wol2] S. Wolpert. Chern forms and the Riemann tensor for the moduli space of curves. Invent. Math. 85(1986), 119-145. MR 87j:32070

[Wol3] S. Wolpert. Geodesic length functions and the Nielsen problem. J. Diff. Geom. 25(1987), 275-296. MR 88e:32032

[Wol4] S. Wolpert. The hyperbolic metric and the geometry of the universal curve. J. Diff. Geom. 31(1990), 417-472. MR 91a:32030

[Wol5] S. Wolpert. The geometry of the Weil-Petersson completion of Teichmüller space. Preprint (2002).

Mathematics Department, University of Chicago, 5734 S. University Avenue, Chicago, ILLINOIS 60637

E-mail address: brock@math.uchicago.edu 